\title{
The effects of shockwave therapy on musculoskeletal conditions based on changes in imaging: a systematic review and meta-analysis with meta-regression
}

Hani Al-Abbad ${ }^{1,2}$, Sophie Allen ${ }^{1}$, Susan Morris ${ }^{1}$, Jackie Reznik ${ }^{3}$, Erik Biros ${ }^{4}$, Bruce Paulik ${ }^{5}$ and Anthony Wright ${ }^{*}$

\begin{abstract}
Background: Shockwave therapy (SWT) is a commonly used intervention for a number of musculoskeletal conditions with varying clinical outcomes. However, the capacity of SWT to influence pathophysiological processes and the morphology of affected tissues remains unclear. The objective of the current review is to evaluate changes in imaging outcomes of musculoskeletal conditions following SWT.

Methods: A comprehensive search of Medline, Embase, Cochrane Controlled Trials Register, CINAHL and SportDiscus was conducted from inception to October 2018. Prospective clinical trials evaluating the effectiveness of SWT based on changes in imaging outcomes were eligible for inclusion. Articles were evaluated independently for risk of bias using the Cochrane Risk of Bias list and the Methodological Index for Non-Randomized Studies. Random-effects meta-analysis and meta-regression with a priori determined covariates was conducted for each condition to determine potential predictors of SWT effects.

Results: Sixty-three studies were included, with data from 27 studies available for effect size pooling. Meta-analyses and meta-regression on imaging outcomes were performed for rotator cuff calcific tendinitis $(n=11)$, plantar fasciitis $(n=7)$ and osteonecrosis of the femoral head $(n=9)$. There was an overall reduction in the size of measured lesion following SWT (MD $8.44 \mathrm{~mm}(95 \% \mathrm{Cl}$ 4.30, 12.57), $p<0.001)$ for calcium deposit diameter, (MD 0.92 $\mathrm{mm}(95 \% \mathrm{Cl} 0.03,1.81), p=0.04)$ for plantar fascia thickness and (MD 4.84\% $(95 \% \mathrm{Cl}-0.06,9.75), p=0.05)$ for lesion size in femoral head osteonecrosis. Meta-regression showed no influence of SWT dosage parameters, however, baseline lesion size was an independent predictor for changes in imaging outcomes.

Conclusions: SWT altered the morphology of musculoskeletal conditions, potentially reflecting changes in underlying pathophysiological processes. The parameters of SWT dosage are not significant predictors of changes in imaging outcomes. Lack of adequate reporting of imaging outcomes limited the conclusions that could be drawn from the current review. Registration number: PROSPERO CRD42018091140.
\end{abstract}

Keywords: Extracorporeal shockwave therapy, Imaging, Systematic review, Meta-analysis

\footnotetext{
* Correspondence: t.wright@curtin.edu.au

'School of Physiotherapy and Exercise Science, Curtin University, GPO Box U1987, Perth, WA, Australia

Full list of author information is available at the end of the article
}

(c) The Author(s). 2020 Open Access This article is licensed under a Creative Commons Attribution 4.0 International License, which permits use, sharing, adaptation, distribution and reproduction in any medium or format, as long as you give appropriate credit to the original author(s) and the source, provide a link to the Creative Commons licence, and indicate if changes were made. The images or other third party material in this article are included in the article's Creative Commons licence, unless indicated otherwise in a credit line to the material. If material is not included in the article's Creative Commons licence and your intended use is not permitted by statutory regulation or exceeds the permitted use, you will need to obtain permission directly from the copyright holder. To view a copy of this licence, visit http://creativecommons.org/licenses/by/4.0/ The Creative Commons Public Domain Dedication waiver (http://creativecommons.org/publicdomain/zero/1.0/) applies to the data made available in this article, unless otherwise stated in a credit line to the data. 


\section{Background}

Shockwave Therapy (SWT) is used to treat a range of musculoskeletal conditions. Focused and radial shockwave (radial pressure waves) are two technically distinct forms of SWT. It has been argued that focused shockwave therapy and radial shockwave therapy should be viewed as distinctly different therapeutic modalities [1]. However, despite the differences in their physical characteristics, method of energy generation and shockwave propagation, focused and radial shockwave types share common clinical indications [2]. SWT is often indicated as a secondary conservative treatment choice for recalcitrant musculoskeletal conditions, unresponsive to standard care $[1,3]$. These indications include plantar fasciitis, Achilles tendinopathy, patellar tendinopathy, calcific and non-calcific shoulder tendinopathy and lateral epicondylitis. Also, bone and cartilage related disorders such as non-union of fractures, osteonecrosis of the femoral head and knee osteoarthritis related bone marrow edema (BME) are among the range of SWT clinical indications. Research evidence for SWT clinical effectiveness varies across the indicated conditions. Good evidence based on systematic reviews exists to support the use of SWT for calcific tendinopathy of the shoulder [4], Achilles tendinopathy [5, 6], knee osteoarthritis [7], early stage osteonecrosis of the femoral head [8] and plantar fasciitis [9]. However, research evidence for the effectiveness of SWT in lateral epicondylitis is variable [1] and is lacking for patellar tendinopathy $[3,6]$.

Focused shockwaves are generated through three mechanisms: electrohydraulic, piezoelectric or electromagnetic methods that convert electrical energy into kinetic energy, whilst radial shockwaves are generated pneumatically [2]. The proposed mechanism of action for SWT is based on mechano-transduction [10]. The delivery of mechanical acoustic energy to the target tissue induces molecular, cellular and tissue responses [11]. Based on animal studies, SWT promotes the expression of various angiogenic and osteogenic growth factors such as vascular endothelial growth factor (VEGF) and bone morphogenetic protein (BMP), which promotes tissue regeneration [12]. In addition, SWT has an anti-inflammatory effect by modulating the expression of interleukins (IL-6 and IL-10) and other cytokines [13].

Clinical outcomes such as pain rating and functional disability scores are commonly used to evaluate the effectiveness of SWT. However, the utilization of objective outcome measures that evaluate changes in the affected musculoskeletal tissues is required to provide evidence of SWT influence on pathophysiological processes in humans. Different imaging techniques are used clinically to establish a diagnosis, guide the delivery of an intervention or to evaluate the effectiveness of an intervention. A range of imaging modalities including magnetic resonance imaging (MRI), ultrasonography (US), Computed Tomography (CT), dual-energy x-ray absorptiometry (DEXA) or plain radiography have been used to support diagnosis and in some cases to evaluate outcomes in studies investigating SWT. Despite lack of consistency in utilization, these imaging modalities are valuable tools for evaluating the morphological characteristics of injured tissues. That can then be used clinically to monitor improvements in the underlying pathophysiological process following an intervention. Therefore, the utilization of suitable imaging tools at appropriate time points as an adjunct to clinical examination may provide a better understanding of the tissue pathophysiology and support the management planning process. Little is known about the capacity of SWT to induce positive improvements in pathophysiological processes in musculoskeletal disorders, as indicated by changes in imaging parameters and no systematic reviews have been conducted on this topic. Therefore, the primary aim of this review was to explore the available evidence from clinical prospective trials with regard to any changes in the morphology of musculoskeletal lesions following SWT, as measured by imaging parameters. The secondary aim was to investigate significant predictors for the SWT effects using meta-regression. We also sought to make recommendations for future research studies.

\section{Methods}

\section{Protocol and registration}

This systematic review and meta-analysis was conducted following the guidelines of the Preferred Reporting Items for Systematic Reviews and Meta-analyses (PRISMA). The protocol of the current review was registered on the International Prospective Register of Systematic Reviews (PROSPERO; 2018, CRD42018091140).

\section{Search strategy}

A comprehensive search using the electronic databases of MEDLINE, EMBASE, CINAHL, SPORTDiscuss and the Cochrane Controlled Trials Register using the PICOS format (population, intervention/exposure, comparison, outcome and study design) was conducted in October 2018. A range of keywords integrated with subject headings relevant to the review were systematically searched to maximize the search results. Keywords for the population category contained terms related to musculoskeletal conditions such as arthritis, fractures, bone marrow edema and tendinopathies. The intervention category contained keywords related to shockwave therapy. The outcome category contained terms related to different imaging methods (e.g. X-ray, MRI, Ultrasound imaging). No specific comparator was added as the primary aim of the review was to examine post intervention imaging changes. An example of the basic search strategy for databases is presented in Additional file 1 .

Eligibility criteria included prospective study designs to avoid any potential selection bias inherent in retrospective studies, adult participants of both genders with an established musculoskeletal diagnosis and reported 
pre and post imaging measures to facilitate evaluation of changes in affected tissue morphology following SWT of any type (focused and radial). Non-human and nonEnglish language studies were excluded.

\section{Study identification}

The principal author conducted the database search. Study screening was carried out using the Covidence platform [14]. Two independent reviewers (HA and SA) screened titles and abstracts to determine eligibility for inclusion. The decision to include or exclude studies, based on the eligibility criteria, was made independently by each author. Full-texts of potentially relevant studies were retrieved for further assessment. Discrepancies in opinions were resolved by a third senior reviewer (AW).

\section{Risk of bias assessment}

The quality of studies was independently evaluated by two reviewers (HA and SA). A third senior reviewer (AW) was involved to resolve differences in assessment. Randomized Controlled Trials (RCTs) were evaluated using the Cochrane Risk of Bias list [15]. The tool evaluates the risk of bias in five domains (selection, performance, attrition, reporting and other) for seven elements to be judged as high, unclear or low risk. A three-point scale was used to assign each degree of risk a number value with higher numbers indicating a lower risk of bias (low risk $=2$, unclear risk $=1$ and high risk $=0$ ). The maximum score of 14 indicates the lowest risk of bias for a given study.

The Methodological Index for Non-Randomized Studies (MINORS) tool $[16,17]$ was used to evaluate the non-randomized studies. The MINORS is a valid and reliable tool that contains 12 methodological items, the first eight items measure attributes specific to noncomparative studies. Four additional items are evaluated only for studies with comparative groups. A higher score indicates a lower risk of bias.

The risk of bias was assessed mainly at the reported measured imaging outcomes level. The score for each risk of bias tool was divided by the total number of points possible to calculate the risk of bias percentage for each study.

\section{Data extraction}

One reviewer (HA) extracted relevant data from individual studies and the data were independently checked by a second reviewer (SA) for consistency. Extracted data included study characteristics such as study design, participant numbers and demographics, the condition treated, parameters and dosage of the SWT intervention (level of energy, number of shocks and sessions), followup period, imaging modality description and findings. Authors were contacted when relevant outcomes were incompletely reported and were asked to provide missing information. When the values of standard deviations
(SD) were not provided for the intervention or control group, these were calculated from confidence intervals, and $p$-values for differences in means, or for group means using the RevMan calculator [18].

\section{Primary outcome}

Changes in measures derived from imaging methods such as MRI, ultrasonography, CT, DEXA or plain radiography reflecting morphological changes in affected musculoskeletal tissues following SWT were the primary evaluated outcomes for this review. The measures of effect were pre to post-imaging changes, demonstrating presence, grade, signal intensity or size of the tissue lesion. The strategy for data synthesis employed a quantitative method in the form of meta-analysis depending upon the type of data extracted, alongside narrative qualitative synthesis. The method of evaluation for each study is clearly stated in the relevant tables.

\section{Statistical analysis}

Quantitative data analysis was performed using RevMan [18]. Meta-analysis was conducted to calculate pooled effect size for included studies. Continuous data were presented as mean differences (MD) and dichotomous data with odds ratios (OR), including the corresponding 95\% confidence interval (CI). Pooling was performed using a random effects model to provide summary effect size owing to expected clinical and methodological heterogeneity. Subgroup analyses were performed separately for each musculoskeletal condition based on SWT type or dosage, control group or use of imaging guidance. Heterogeneity was assessed statistically with $\chi^{2}$ test and $\mathrm{I}^{2}$ statistics and significance was set at $p<0.05$. Meta-regression was carried out to explain the source of heterogeneity based on important mediating covariates such as SWT dose parameters, baseline lesion size, utilization of anesthesia and imaging guidance (determined a priori).

\section{Results}

\section{Study selection and characteristics}

The database searches resulted in 789 titles. (Fig. 1). Following the removal of duplicates and exclusion of records based on title and abstract screening, a total of 93 studies were available for full-text review. A total of 30 studies were further excluded with inappropriate study design (predominantly retrospective studies) or lack of imaging outcome as the most frequent reasons for exclusion, leaving a final selection of 63 studies meeting the inclusion criteria. Of the included studies, 30 used an RCT design and the remaining 33 studies were prospective cohort trials. 


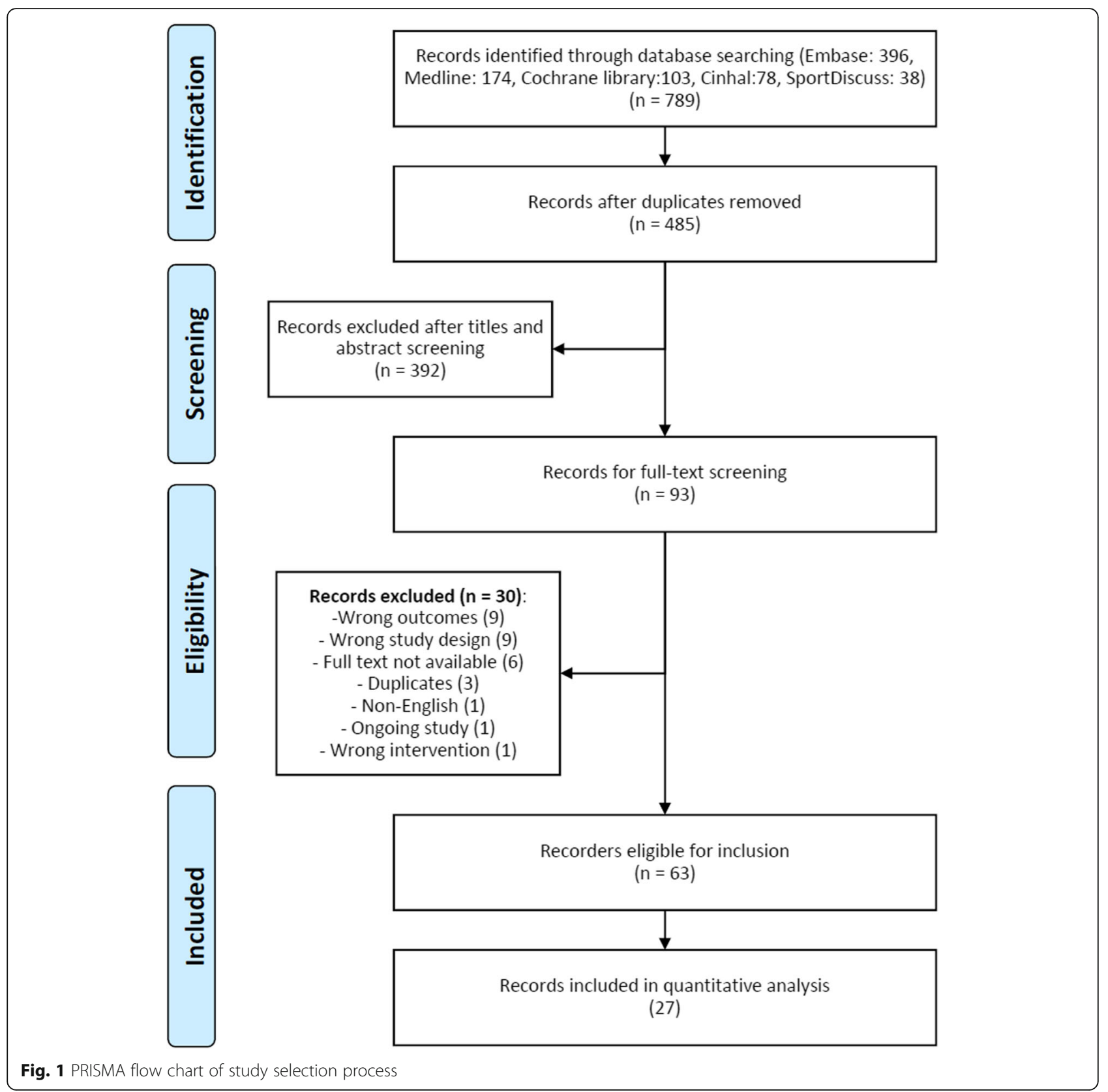

\section{Description of included studies}

The total number of participants in the SWT groups (focused and radial SWT) was 3110. The most commonly evaluated musculoskeletal condition was rotator cuff calcific tendinitis (23 studies), followed by plantar fasciitis (13 studies), femoral head necrosis (12 studies) and fracture nonunion (4 studies). There were a few other individual studies evaluating different musculoskeletal conditions (Fig. 2).

\section{Quality assessment}

The risk of bias assessment is summarized in Additional file 2 for included RCTs and prospective trials respectively. The mean score (\%) \pm SD of the Cochrane risk of bias tool among the 31 RCT studies was $59.68 \pm 18.95$, while the mean score was $73.61 \pm 7.65$ for non-RCT studies according to the MINORS tool. Items of risk of bias rating across all included RCT and non-RCT studies are shown in Additional file 2. Inter-rater reliability of the risk of bias assessment was calculated using the kappa coefficient $(\mathrm{k})$. The overall agreement between the two primary reviewers was $79.84 \%(\mathrm{k}=0.53)$ indicating moderate agreement.

\section{Rotator cuff calcific tendinitis}

There were 23 studies (8 RCTs) published between 1995 and 2017 evaluating the effect of SWT on rotator cuff calcific tendinitis. The total sample was 1110 (1141 


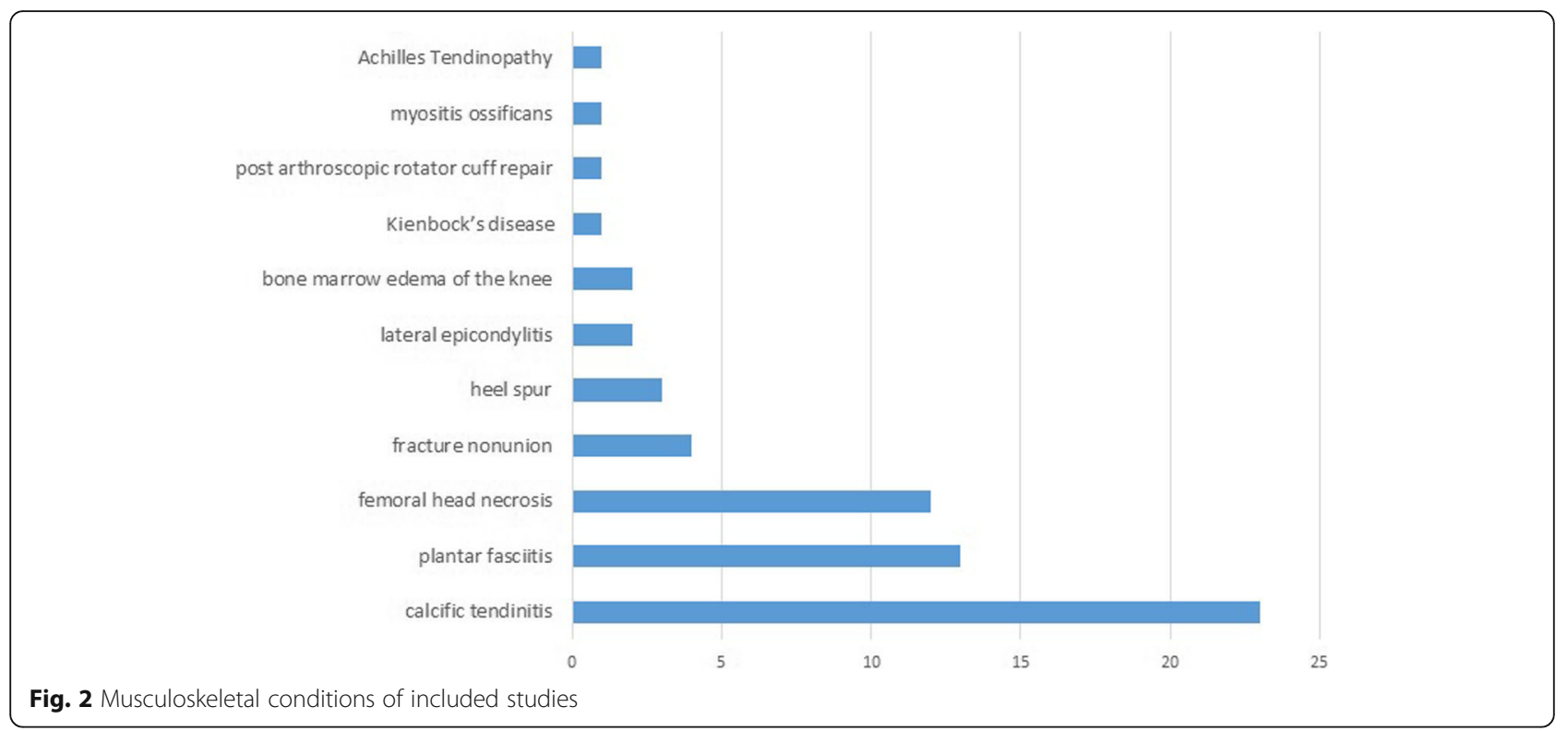

shoulders) participants. The main imaging inclusion criterion for participant recruitment was the presence of symptomatic type I or II calcification of the rotator cuff based on the Gartner and Simons radiographic classification [19], as detected on radiographs. Detection of glenohumeral or acromioclavicular arthritis, rotator cuff tear or type III calcification were the main exclusion criteria.

Focused type SWT was used in 21 studies, while two studies used radial type SWT. The mean \pm SD SWT delivered shocks was $2104 \pm 990.57(1000-6000)$ and the mean energy flux density (EFD) was $0.26 \pm 0.15(0.02-0.6) \mathrm{mJ} /$ $\mathrm{mm}^{2}$. The mean number of SWT sessions was $2.66 \pm 1.91$ (1-8) with $10.1 \pm 7.26(1-35)$ days interval between sessions. Ten studies used anesthesia prior to SWT application and 14 studies used imaging guidance to target calcium deposits. Further details on included studies characteristics and SWT parameters are presented in Table 1.

Plain radiographs obtained at different time points post SWT was the method used to assess imaging outcomes in 21 studies, while two studies used ultrasonography. Four studies reported the change in calcium deposit diameter $(\mathrm{mm}), 16$ studies reported the number of participants demonstrating total calcification resorption and three reported on reduction or fragmentation of the calcium deposit (Table 2).

The size of calcium deposit was shown to reduce following SWT application (MD $8.44 \mathrm{~mm}$ (95\%CI 4.30, 12.57), $p<0.001$; Fig. 3) within a period of 1 week [21], 3 months [36] and 12 months [26, 29, 31]. Baseline calcium deposit size was the only covariate to explain the variance related to the effect of SWT (Coeff. $1.38 \mathrm{~mm}$ $(95 \%$ CI $0.98,1.77) \mathrm{I}^{2}=43.55 \%$, Adj. $\mathrm{R}^{2}=99.18 \%, p=$ 0.002). No variables related to SWT treatment parameters were significant covariates.
The reduction in calcium deposit size favored SWT compared to placebo but did not reach statistical significance (MD - $11.26 \mathrm{~mm}(95 \% \mathrm{CI}-24.68,2.17), P=0.1)$ $[21,29]$. However, the effect of SWT on calcium deposit size was less compared to ultrasound-guided needling (MD 4.25 (95\%CI 2.27, 6.24), $p=0.006$ ), (Fig. 4) [26, 31]. Total calcium resorption was reported in $222 / 559$ (35\%) shoulders. Total calcium resorption was greater in the SWT group compared to placebo (OR 6.38 (95\%CI 1.33, 30.70, $p=0.02$ ), but not compared to ultrasound guidedneedling (OR 0.27 (95\%CI 0.12, 0.64), $p=0.003$ ), (Fig. 5).

\section{Plantar fasciitis and heel spurs}

A total of 13 studies (7 RCTs) published between 2008 and 2018 evaluated imaging changes in plantar fasciitis following SWT. The total number of included participants was 359 (365 heels) diagnosed with symptomatic heel pain in addition to imaging features of plantar fascia thickening $(>4 \mathrm{~mm})$ as the main inclusion criteria. The main exclusion criteria were the presence of systemic disease such as rheumatoid arthritis, diabetes mellitus, vascular abnormalities or neurological impairments.

Eight studies utilized focused-SWT, radial-SWT was used in three studies and one study employed a combined focused and radial SWT type stimulus. The type of SWT was not reported in one study [43]. The mean SWT shocks per treatment session was $2426.92 \pm 965.36$ (1000-4000) with a mean EFD of $0.22 \pm 0.14(0.03-0.42)$ $\mathrm{mJ} / \mathrm{mm}^{2}$. The mean number of treatment sessions was $3.04 \pm 1.67(1-8)$ sessions with a mean of $24.79 \pm 43.56$ (1-140) days between sessions. Only two studies used anesthesia before the application of SWT and four studies employed sonographic guidance to localize the area of application (Table 3). 
Table 1 Characteristics of studies and intervention details for rotator cuff calcifying tendinitis

\begin{tabular}{|c|c|c|c|c|c|c|c|c|c|}
\hline Author (year) & Study design & N & $\begin{array}{l}\text { Mean } \\
\text { age } \pm S D \\
\text { or } \\
\text { (range) }\end{array}$ & $\begin{array}{l}\text { Mean } \\
\text { symptoms } \\
\text { duration } \pm \\
\text { SD or } \\
\text { (range), } \\
\text { months }\end{array}$ & Area of SWT application & $\begin{array}{l}\text { Dosage in } \\
\text { impulses*EFD } \\
\left(\mathrm{mJ} / \mathrm{mm}^{2}\right) / \text { bar }\end{array}$ & $\begin{array}{l}\text { No. of } \\
\text { sessions }\end{array}$ & $\begin{array}{l}\text { Interval } \\
\text { between } \\
\text { sessions }\end{array}$ & $\begin{array}{l}\text { Co- } \\
\text { intervention, } \\
\text { anesthesia }\end{array}$ \\
\hline $\begin{array}{l}\text { Albert (2007) } \\
\text { [20] }\end{array}$ & $\mathrm{RCT}$ & $\begin{array}{l}40 \\
\text { each } \\
\text { group }\end{array}$ & $\begin{array}{l}\text { High } \\
\text { SWT: } \\
46.6 \text { (31- } \\
64) \\
\text { LoW } \\
\text { SWT: } \\
47.5 \text { (32- } \\
69)\end{array}$ & $\begin{array}{l}\text { High SWT: } \\
41.2 \text { (6- } \\
\text { 120) } \\
\text { LoW SWT: } \\
\text { 36.4 (7- } \\
160)\end{array}$ & $\begin{array}{l}\text { Calcific deposit was identified } \\
\text { using fluoroscopy }\end{array}$ & $\begin{array}{l}\text { High SWT: } \\
2000^{*} 0.45 \\
\text { LoW SWT: } \\
2000^{*} 0.02\end{array}$ & 2 & 14 days & None, yes \\
\hline $\begin{array}{l}\text { Cacchio } \\
(2006)[21]\end{array}$ & RCT & 45 & $\begin{array}{l}56.12 \pm \\
1.98\end{array}$ & $14 \pm 4.95$ & $\begin{array}{l}\text { Seated with shoulder abducted } \\
\text { at } 45^{\circ} \text { in external rotation, } \\
\text { elbow at } 90^{\circ} \text {, SWT was placed } \\
\text { in the direction of calcifications. }\end{array}$ & $\begin{array}{l}2500^{*} 0.1 \\
\text { pressure of } \\
2.5 \text { bar }\end{array}$ & 4 & 1 week & None \\
\hline $\begin{array}{l}\text { Charrin } \\
\text { (2001) [22] }\end{array}$ & $\begin{array}{l}\text { Prospective } \\
\text { open design }\end{array}$ & 32 & $\begin{array}{l}49.8 \pm \\
5.9\end{array}$ & $52.1 \pm 48.5$ & $\begin{array}{l}\text { Ultrasound was used to identify } \\
\text { the lesions and aimed at the } \\
\text { calcific deposit at all times. }\end{array}$ & $2000 * 0.32$ & 2 or 3 & $\begin{array}{l}13.4 \pm \\
6.4 \text { days }\end{array}$ & None \\
\hline $\begin{array}{l}\text { Cosentino } \\
\text { (2003) [23] }\end{array}$ & RCT & 35 & 51.8 & $15(10-20)$ & $\begin{array}{l}\text { SWT was placed in the direction } \\
\text { of the calcification based on } \\
\text { sonographic examination }\end{array}$ & $1200 * 0.28$ & 4 & 4-7 days & None \\
\hline $\begin{array}{l}\text { Daecke } \\
\text { (2002) [24] }\end{array}$ & $\begin{array}{l}\text { Prospective } \\
\text { comparative } \\
\text { design }\end{array}$ & $\begin{array}{l}\text { Group } \\
\text { A: } 56 \\
\text { Group } \\
\text { B: } 59\end{array}$ & $\begin{array}{l}49(28- \\
77)\end{array}$ & $\begin{array}{l}5(1-36) \\
\text { years }\end{array}$ & $\begin{array}{l}\text { SWT was performed after } \\
\text { localization of the calcification in } \\
2 \text { planes by fluoroscopy }\end{array}$ & $2000 * 0.3$ & $\begin{array}{l}\text { Group } \\
\text { A: } 1 \\
\text { Group } \\
\text { B: } 2\end{array}$ & 1 week & None, yes \\
\hline $\begin{array}{l}\text { DeBoer } \\
(2017)[25]\end{array}$ & RCT & 14 & $\begin{array}{l}53 \\
(95 \% \mathrm{Cl} \\
48,58)\end{array}$ & $>6$ & NR & $\begin{array}{l}2500^{*} 0.1 \\
\text { pressure of } \\
2.5 \text { bar }\end{array}$ & 5 & 1 week & None \\
\hline $\begin{array}{l}\text { DelCastillo- } \\
\text { Gonzalez } \\
\text { (2016) [26] }\end{array}$ & RCT & 80 & $49 \pm 7$ & NR & $\begin{array}{l}\text { The calcification was identified } \\
\text { by fluoroscopy in seated } \\
\text { position }\end{array}$ & $2000^{*} 0.2$ & 8 & $\begin{array}{l}\text { Twice } \\
\text { weekly }\end{array}$ & None \\
\hline $\begin{array}{l}\text { Farr (2011) } \\
{[27]}\end{array}$ & RCT & 15 & $49.7 \pm 9$ & $>6$ & $\begin{array}{l}\text { The calcific deposit was located } \\
\text { by fluoroscopy. The computer } \\
\text { calculated angle and distance } \\
\text { for maximum precision. }\end{array}$ & $\begin{array}{l}\text { Group A: } \\
3200^{*} 0.3 \\
\text { Group B: } \\
1600^{*} 0.2\end{array}$ & $\begin{array}{l}\text { Group } \\
\text { A: } 1 \\
\text { Group } \\
\text { B: } 2\end{array}$ & 1 week & None, yes \\
\hline $\begin{array}{l}\text { Gerdesmeyer } \\
\text { (2003) [28] }\end{array}$ & $\mathrm{RCT}$ & $\begin{array}{l}48 \\
\text { each }\end{array}$ & $\begin{array}{l}\text { High: } \\
51.6 \pm \\
8.5 \\
\text { Low: } \\
47.3 \pm \\
8.5 \\
\text { placebo: } \\
52.3 \pm \\
9.8\end{array}$ & $\begin{array}{l}\text { High: } \\
42.6 \pm 23.2 \\
\text { Low: } \\
42.8 \pm 25.2 \\
\text { placebo: } \\
41.3 \pm 28.6\end{array}$ & $\begin{array}{l}\text { Using fluoroscopy in prone } \\
\text { position as the shoulder was } \\
\text { rotated until the calcific deposit } \\
\text { was identified }\end{array}$ & $\begin{array}{l}\text { High: } \\
1500^{*} 0.32 \\
\text { Low: } \\
6000^{*} 0.08\end{array}$ & 2 & 14 days & $\begin{array}{l}\text { All groups } \\
\text { received } 10 \\
\text { physiotherapy } \\
\text { sessions after } \\
\text { SWT, no }\end{array}$ \\
\hline $\begin{array}{l}\text { Hsu (2008) } \\
{[29]}\end{array}$ & RCT & 33 & $\begin{array}{l}54.4(30- \\
70)\end{array}$ & $12.3(6-72)$ & NR & $1000 * 0.55$ & 2 & 14 days & None, yes \\
\hline $\begin{array}{l}\text { Jakobeit } \\
\text { (2002) [30] }\end{array}$ & & 80 & 53.3 & $>6$ & $\begin{array}{l}\text { SWT was performed with } \\
\text { retroversion and adduction of } \\
\text { the shoulder as far as possible } \\
\text { under ultrasound monitoring }\end{array}$ & $1800 * 0.42$ & $1-5$ & $\begin{array}{l}4-6 \\
\text { weeks }\end{array}$ & None, yes \\
\hline $\begin{array}{l}\text { Kim }(2014) \\
{[31]}\end{array}$ & RCT & 29 & $\begin{array}{l}57.4(47- \\
78)\end{array}$ & $>3$ & $\begin{array}{l}\text { SWT was performed in the } \\
\text { sitting position by aiming at the } \\
\text { maximum sore spot according } \\
\text { to anatomic targeting }\end{array}$ & $1000 * 0.36$ & 3 & 1 week & NSAIDs, no \\
\hline $\begin{array}{l}\text { Kransy (2005) } \\
{[32]}\end{array}$ & $\mathrm{RCT}$ & 40 & $\begin{array}{l}49.4 \\
(32.4- \\
63.5)\end{array}$ & $\begin{array}{l}30.5(12- \\
60)\end{array}$ & $\begin{array}{l}\text { In prone position, the calcific } \\
\text { deposit had been positioned in } \\
\text { the center of the scan unit. }\end{array}$ & $2500 * 0.36$ & 1 & NA & None, yes \\
\hline $\begin{array}{l}\text { Loew (1999) } \\
\text { [33] }\end{array}$ & RCT & $\begin{array}{l}20 \\
\text { each }\end{array}$ & $\begin{array}{l}46(28- \\
77)\end{array}$ & 36 & $\begin{array}{l}\text { The calcification was visualized } \\
\text { using fluoroscopy before and at }\end{array}$ & $\begin{array}{l}\text { Group 1: } \\
2000^{*} 0.1\end{array}$ & $\begin{array}{l}\text { Group } \\
1,2: 1\end{array}$ & 1 week & None, yes \\
\hline
\end{tabular}


Table 1 Characteristics of studies and intervention details for rotator cuff calcifying tendinitis (Continued)

\begin{tabular}{|c|c|c|c|c|c|c|c|c|c|}
\hline Author (year) & Study design & $\mathrm{N}$ & $\begin{array}{l}\text { Mean } \\
\text { age } \pm S D \\
\text { or } \\
\text { (range) }\end{array}$ & $\begin{array}{l}\text { Mean } \\
\text { symptoms } \\
\text { duration } \pm \\
\text { SD or } \\
\text { (range), } \\
\text { months }\end{array}$ & Area of SWT application & $\begin{array}{l}\text { Dosage in } \\
\text { impulses*EFD } \\
\left(\mathrm{mJ} / \mathrm{mm}^{2}\right) / \text { bar }\end{array}$ & $\begin{array}{l}\text { No. of } \\
\text { sessions }\end{array}$ & $\begin{array}{l}\text { Interval } \\
\text { between } \\
\text { sessions }\end{array}$ & $\begin{array}{l}\text { Co- } \\
\text { intervention, } \\
\text { anesthesia }\end{array}$ \\
\hline & & & & & intervals during treatment. & $\begin{array}{l}\text { Group 2,3: } \\
2000 * 0.3\end{array}$ & $\begin{array}{l}\text { Group } \\
3: 2\end{array}$ & & \\
\hline $\begin{array}{l}\text { Lowe (1995) } \\
\text { [34] }\end{array}$ & $\begin{array}{l}\text { Prospective } \\
\text { open design }\end{array}$ & 20 & $\begin{array}{l}50(35- \\
72)\end{array}$ & $>12$ & $\begin{array}{l}\text { SWT was performed as } \\
\text { localization of the calcium } \\
\text { deposit was achieved with an } \\
\text { image intensifier that was } \\
\text { adjusted automatically in two } \\
\text { planes. The head was placed in } \\
\text { a ventro-lateral position }\end{array}$ & $\begin{array}{l}2000^{*} 18-22 \\
\mathrm{kV}\end{array}$ & 2 & 2 weeks & None, yes \\
\hline $\begin{array}{l}\text { Moretti } \\
\text { (2005) [35] }\end{array}$ & $\begin{array}{l}\text { Prospective } \\
\text { open design }\end{array}$ & 54 & $\begin{array}{l}43(34- \\
66)\end{array}$ & $>3$ & NR & $2500 * 0.11$ & 4 & 3 days & None \\
\hline $\begin{array}{l}\text { Pan (2003) } \\
{[36]}\end{array}$ & $\mathrm{RCT}$ & 32 & $\begin{array}{l}55.21 \pm \\
2.01\end{array}$ & $\begin{array}{l}24.55 \pm \\
6.45\end{array}$ & $\begin{array}{l}\text { SWT was positioned at the } \\
\text { marked painful area as defined } \\
\text { by sonography before each } \\
\text { treatment }\end{array}$ & $\begin{array}{l}2000^{*} 0.26- \\
0.32\end{array}$ & 2 & 14 days & None \\
\hline $\begin{array}{l}\text { Pigozzi (2000) } \\
\text { [37] }\end{array}$ & $\begin{array}{l}\text { Prospective } \\
\text { open design }\end{array}$ & 19 & $\begin{array}{l}38(18- \\
69)\end{array}$ & $>2$ & NR & $2000 * 0.21$ & 8 & 1 week & None \\
\hline $\begin{array}{l}\text { Pleiner (2004) } \\
\text { [38] }\end{array}$ & $\mathrm{RCT}$ & $23(31)$ & $54 \pm 11$ & $>6$ & $\begin{array}{l}\text { SWT was focused on the point } \\
\text { of maximum pain }\end{array}$ & $2000 * 0.28$ & 2 & 14 days & None \\
\hline $\begin{array}{l}\text { Rompe } \\
\text { (1995) [39] }\end{array}$ & $\begin{array}{l}\text { Prospective } \\
\text { open design }\end{array}$ & 40 & 47 & $\begin{array}{l}25(12- \\
120)\end{array}$ & $\begin{array}{l}\text { SWT was administered once the } \\
\text { calcium deposit is situated in } \\
\text { the center of the C-arm. }\end{array}$ & $1500 * 0.28$ & 1 & NA & None, yes \\
\hline $\begin{array}{l}\text { Rompe } \\
\text { (2001) [40] }\end{array}$ & $\begin{array}{l}\text { prospective } \\
\text { quasi- } \\
\text { randomized }\end{array}$ & 50 & $\begin{array}{l}49.6 \pm \\
7.5\end{array}$ & $52.6 \pm 54.4$ & $\begin{array}{l}\text { SWT was administered once the } \\
\text { calcium deposit is situated in } \\
\text { the center of the C-arm }\end{array}$ & $3000 * 0.6$ & 1 & NA & $\begin{array}{l}\text { Active exercise } \\
\text { for } 4 \text { to } 6 \\
\text { weeks, yes }\end{array}$ \\
\hline $\begin{array}{l}\text { Sabeti- } \\
\text { Aschraf } \\
\text { (2005) [41] }\end{array}$ & $\mathrm{RCT}$ & $\begin{array}{l}25 \\
\text { each }\end{array}$ & $\begin{array}{l}52.68 \pm \\
8.19\end{array}$ & $>6$ & $\begin{array}{l}\text { In group 1, the angle and } \\
\text { distance between the SWT and } \\
\text { shoulder were adjusted until the } \\
\text { patient reported to the point of } \\
\text { maximum tenderness. In group } \\
\text { 2, the Lithotrack device was } \\
\text { used to locate the calcium } \\
\text { deposit in the center of a } \\
\text { crosshairs by fluoroscopy in } 2 \\
\text { planes. The computer calculated } \\
\text { the angle and distance to } \\
\text { provide maximum precision. }\end{array}$ & $1000 * 0.08$ & 3 & 1 week & None \\
\hline $\begin{array}{l}\text { Tornese } \\
\text { (2011) [42] }\end{array}$ & $\mathrm{RCT}$ & 35 & 52.6 & NR & $\begin{array}{l}\text { Group A (Neutral position): the } \\
\text { subject lay supine with shoulder } \\
\text { in neutral rotation, the arm } \\
\text { placed alongside the trunk and } \\
\text { the hand resting on the } \\
\text { abdomen } \\
\text { Group B (Hyperextended } \\
\text { internal rotation): the subject lay } \\
\text { supine with shoulder in } \\
\text { hyperextension and internal } \\
\text { rotation with the hand placed } \\
\text { under the buttock and the palm } \\
\text { facing down }\end{array}$ & $1800 * 0.22$ & 3 & 1 week & None \\
\hline
\end{tabular}


Table 2 Imaging outcome measures for rotator cuff calcifying tendinitis

\begin{tabular}{|c|c|c|c|c|c|c|c|}
\hline \multirow[t]{3}{*}{ Author (year) } & \multirow{3}{*}{$\begin{array}{l}\text { SWT } \\
\text { type }\end{array}$} & \multirow[t]{3}{*}{ Comparator } & \multirow[t]{3}{*}{ Imaging outcome } & \multicolumn{4}{|l|}{ Follow-up } \\
\hline & & & & \multirow[t]{2}{*}{ Period } & \multirow{2}{*}{$\begin{array}{l}\text { Baseline - F/U } \\
\text { Mean } \pm S D\end{array}$} & \multicolumn{2}{|l|}{$\boldsymbol{P}$ value } \\
\hline & & & & & & $\begin{array}{l}\text { Within } \\
\text { group }\end{array}$ & $\begin{array}{l}\text { Between } \\
\text { group }\end{array}$ \\
\hline $\begin{array}{l}\text { Albert (2007) } \\
\text { [20] }\end{array}$ & $\begin{array}{l}\text { High } \\
\text { SWT }\end{array}$ & LoW SWT & $\begin{array}{l}\text { The radiological aspects of } \\
\text { calcifications (i.e. type, size and } \\
\text { location) were determined through } \\
\text { lateral and anteroposterior } \\
\text { shoulder views in neutral, external } \\
\text { and internal rotation. Changes } \\
\text { were graded as no resorption, } \\
\text { partial resorption and total or } \\
\text { subtotal resorption (over } 80 \% \\
\text { reduction of calcified surface on } \\
\text { anteroposterior view) }\end{array}$ & $\begin{array}{l}3 \\
\text { months }\end{array}$ & $\begin{array}{l}\text { High: } 6(15 \%) \text { had total or subtotal } \\
\text { resorption. } 3(7.5 \%) \text { had partial } \\
\text { resorption } \\
\text { Low: } 2(5 \%) \text { had total or subtotal } \\
\text { resorption. } 5(12.5 \%) \text { had partial } \\
\text { resorption }\end{array}$ & NR & NR \\
\hline $\begin{array}{l}\text { Cacchio } \\
\text { (2006) [21] }\end{array}$ & $\begin{array}{l}\text { R- } \\
\text { SWT }\end{array}$ & Placebo & $\begin{array}{l}\text { The radiological aspects of } \\
\text { calcifications (i.e. type, size and } \\
\text { location) were determined through } \\
\text { lateral and anteroposterior } \\
\text { shoulder views in } 45 \text { degrees of } \\
\text { external and internal rotation were } \\
\text { acquired. Type of calcification was } \\
\text { evaluated according to the Gartner } \\
\text { and Simons classification. A caliper } \\
\text { that evaluated calcification length } \\
\text { (in millimeters) was used for size } \\
\text { measurement. }\end{array}$ & 1 week & $\begin{array}{l}\text { 39(86.6\%) had total resorption, } \\
6(13.4 \%) \text { had partial resorption, } \\
\text { while the control group, no } \\
\text { complete disappearance of } \\
\text { calcifications was observed. } \\
\text { The mean Calcium deposits } \\
\text { diameter (mm) pre-SWT was } \\
21.3 \pm 7.5 \text {, post-SWT was } 0.85 \pm 1.2 \text {. } \\
\text { In contrast, pre-sham was } 19.7 \pm \\
\text { 8.3, post-sham was } 18.85 .8 \pm 6.4 \text {. }\end{array}$ & $<0.00$ & $<0.00$ \\
\hline $\begin{array}{l}\text { Charrin } \\
\text { (2001) [22] }\end{array}$ & $\begin{array}{l}\text { F- } \\
\text { SWT }\end{array}$ & None & $\begin{array}{l}\text { Calcific deposit appearance was } \\
\text { assessed on a plain radiograph in } \\
\text { neutral rotation }\end{array}$ & $\begin{array}{l}3,6,12 \\
24 \\
\text { weeks }\end{array}$ & $\begin{array}{l}\text { After } 12 \text { weeks, } 2 / 30 \text { had total } \\
\text { resorption. } 5 \text { deposits had partial } \\
\text { resorption. } \\
\text { After } 24 \text { weeks, } 5 / 29 \text { had total } \\
\text { resorption and } 2 \text { deposits had } \\
\text { partial resorption }\end{array}$ & NR & NA \\
\hline $\begin{array}{l}\text { Cosentino } \\
\text { (2003) [23] }\end{array}$ & $\begin{array}{l}\text { F- } \\
\text { SWT }\end{array}$ & Placebo & $\begin{array}{l}\text { Variations in the dimension of the } \\
\text { calcification were evaluated by } \\
\text { anteroposterior views. Modification } \\
\text { of the calcification (a reduction of } \\
\text { size of }>2 \mathrm{~mm} \text { ) was indicated as } \\
\text { disintegration; the total } \\
\text { disappearance was indicated as } \\
\text { dissolution. }\end{array}$ & 1 month & $\begin{array}{l}11(31 \%) \text { had total resorption, } \\
14(40 \%) \text { had partial resorption. } \\
\text { Calcification remained unchanged } \\
\text { in the control group }\end{array}$ & $<0.001$ & NR \\
\hline $\begin{array}{l}\text { Daecke } \\
\text { (2002) [24] }\end{array}$ & $\begin{array}{l}\text { F- } \\
\text { SWT }\end{array}$ & 1 vs 2 sessions & $\begin{array}{l}\text { Anteroposterior radiograph in } \\
\text { internal and external rotation was } \\
\text { obtained to show obvious changes } \\
\text { in the shape and structure } \\
\text { (disintegration) or complete } \\
\text { resorption of the calcification }\end{array}$ & $\begin{array}{l}3,6 \\
\text { months, } \\
4 \text { years }\end{array}$ & $\begin{array}{l}30 \% \text { in group A and } 52 \% \text { in group } \\
\text { B had partial or total resorption } \\
\text { after } 3 \text { months, } 47 \text { and } 77 \% \text { after } 6 \\
\text { months and } 93 \% \text { for both groups } \\
\text { at } 4 \text { years }\end{array}$ & NR & $\begin{array}{l}<0.046 \text { at } 6 \\
\text { months }\end{array}$ \\
\hline $\begin{array}{l}\text { DeBoer } \\
\text { (2017) [25] }\end{array}$ & $\begin{array}{l}\text { R- } \\
\text { SWT }\end{array}$ & $\begin{array}{l}\text { Ultrasound } \\
\text { Needling (UN) }\end{array}$ & $\begin{array}{l}\text { Scoring of calcification deposits } \\
\text { was assessed through the Gartner } \\
\text { Classification of Calcific Tendinitis. }\end{array}$ & 6 weeks & $\begin{array}{l}1 / 14(7 \%) \text { in the R-SWT group had } \\
\text { total resorption vs } 5 / 11(45.5 \%) \text { in } \\
\text { UN group }\end{array}$ & NR & 0.029 \\
\hline $\begin{array}{l}\text { DelCastillo- } \\
\text { Gonzalez } \\
(2016)[26]\end{array}$ & $\begin{array}{l}\text { F- } \\
\text { SWT }\end{array}$ & $\begin{array}{l}\text { Ultrasound- } \\
\text { guided } \\
\text { percutaneous } \\
\text { lavage (UGPL) }\end{array}$ & $\begin{array}{l}\text { Calcification size measurement was } \\
\text { assessed by ultrasound imaging. }\end{array}$ & $\begin{array}{l}3,6,12 \\
\text { months }\end{array}$ & $\begin{array}{l}55.6 \% \text { had total resorption by } 12 \\
\text { months in the SWT group vs } \\
86.78 \% \text { in the UGPL group. } \\
\text { The mean Calcium deposits } \\
\text { diameter (mm) pre-SWT was } \\
10.53 \pm 5.29 \text {, post-SWT was } 4.67 \pm \\
6.08 \text { after } 12 \text { months. In contrast, } \\
\text { pre-UGPL was } 12.075 \pm 4.85 \text {, post- } \\
\text { UGPL was } 1.56 \pm 2.79 \text {. }\end{array}$ & $<0.01$ & $<0.01$ \\
\hline $\begin{array}{l}\text { Farr (2011) } \\
{[27]}\end{array}$ & $\begin{array}{l}\text { F- } \\
\text { SWT }\end{array}$ & LoW SWT & $\begin{array}{l}\text { Radiological difference of the } \\
\text { calcific deposit was rated as } \\
\text { improvement, unchanged or } \\
\text { worsening }\end{array}$ & $\begin{array}{l}6,12 \\
\text { weeks }\end{array}$ & $\begin{array}{l}58 \% \text { improved in group A } \\
\text { compared to } 69 \% \text { in group B after } \\
12 \text { weeks. } \\
5 \text { in group A, and } 4 \text { in group B } \\
\text { had total resorption }\end{array}$ & NR & $N R$ \\
\hline
\end{tabular}


Table 2 Imaging outcome measures for rotator cuff calcifying tendinitis (Continued)

\begin{tabular}{|c|c|c|c|c|c|c|c|}
\hline \multirow[t]{3}{*}{ Author (year) } & \multirow{3}{*}{$\begin{array}{l}\text { SWT } \\
\text { type }\end{array}$} & \multirow[t]{3}{*}{ Comparator } & \multirow[t]{3}{*}{ Imaging outcome } & \multicolumn{4}{|c|}{ Follow-up } \\
\hline & & & & \multirow[t]{2}{*}{ Period } & \multirow{2}{*}{$\begin{array}{l}\text { Baseline - F/U } \\
\text { Mean } \pm S D\end{array}$} & \multicolumn{2}{|l|}{$\boldsymbol{P}$ value } \\
\hline & & & & & & $\begin{array}{l}\text { Within } \\
\text { group }\end{array}$ & $\begin{array}{l}\text { Between } \\
\text { group }\end{array}$ \\
\hline $\begin{array}{l}\text { Gerdesmeyer } \\
\text { (2003) [28] }\end{array}$ & $\begin{array}{l}\text { F- } \\
\text { SWT }\end{array}$ & $\begin{array}{l}\text { Low SWT and } \\
\text { placebo }\end{array}$ & $\begin{array}{l}\text { The radiological aspects of } \\
\text { calcifications (i.e. type, size and } \\
\text { location) were determined through } \\
\text { anteroposterior shoulder views in } \\
45 \text { degrees of external and internal } \\
\text { rotation. }\end{array}$ & $\begin{array}{l}3,6,12 \\
\text { months }\end{array}$ & $\begin{array}{l}\text { High: } 60 \% \text { had total resorption } \\
\text { within } 6 \text { months and } 86 \% \text { after } 12 \\
\text { months. Low: } 21 \% \text { had total } \\
\text { resorption within } 6 \text { months and } \\
37 \% \text { after } 12 \text { months. Placebo: } 11 \% \\
\text { had total resorption within } 6 \\
\text { months and } 25 \% \text { after } 12 \text { months. } \\
\text { Calcific deposit size (mm2) mean } \\
\text { change from baseline after } 12 \\
\text { months was }-162.2 \text { ( } 95 \% \mathrm{Cl}-204 \\
\text { to }-120 \text { ) in the High-SWT group, } \\
-91.5 \text { ( } 95 \% \mathrm{Cl}-148 \text { to }-35.1 \text { ) low- } \\
\text { SWT group and - } 46.8 \text { ( } 95 \% \mathrm{Cl} \\
-74.3 \text { to }-19.3 \text { ) in the placebo } \\
\text { group }\end{array}$ & $N R$ & $\begin{array}{l}\text { group } 1 \text { vs } 3 \\
P<0.01 \text {, group } \\
2 \text { vs } 3 P=0.1 \\
\text { group } 1 \text { vs } 2 \\
p=0.04\end{array}$ \\
\hline $\begin{array}{l}\text { Hsu (2008) } \\
{[29]}\end{array}$ & $\begin{array}{l}\text { F- } \\
\text { SWT }\end{array}$ & Placebo & $\begin{array}{l}\text { An anteroposterior radiograph } \\
\text { with the arm in neutral rotation } \\
\text { was obtained. The calcific deposits } \\
\text { were categorized according to } \\
\text { morphology and size (the longest } \\
\text { length of the calcium deposit). } \\
\text { Scoring of calcification deposits } \\
\text { was assessed through the Gartner } \\
\text { Classification of Calcific Tendinitis }\end{array}$ & $\begin{array}{l}6 \text { weeks, } \\
3,6,12 \\
\text { months }\end{array}$ & $\begin{array}{l}7(21.2 \%) \text { had total resorption, } \\
11(36.6 \%) \text { had partial resorption. In } \\
\text { the control group, none had total } \\
\text { resorption and } 2(15.3 \%) \text { had partial } \\
\text { resorption. } \\
\text { The mean Calcium deposits } \\
\text { diameter (mm) pre-SWT was } \\
11.9 \pm 5.4(3.4-23.5) \text {, post-SWT was } \\
5.5 \pm 6.3(0-18.7) \text {. In contrast, pre- } \\
\text { sham was 10.5 } 6.4(2.5-20.4) \text {, } \\
\text { post-sham was } 9.8 \pm 5.9(2.3-21) \text {. }\end{array}$ & $<0.01$ & $<0.01$ \\
\hline $\begin{array}{l}\text { Jakobeit } \\
\text { (2002) [30] }\end{array}$ & $\begin{array}{l}\text { F- } \\
\text { SWT }\end{array}$ & None & $\begin{array}{l}\text { Diagnostic ultrasonography and } \\
\text { radiography were used to classify } \\
\text { the calcareous deposits in } 5 \\
\text { categories according to their } \\
\text { morphological appearance and } \\
\text { size. }\end{array}$ & 4 weeks & $\begin{array}{l}57 / 80(71.25 \%) \text { had total } \\
\text { resorption. 16/80 (20\%) had partial } \\
\text { resorption }\end{array}$ & $N R$ & NA \\
\hline $\begin{array}{l}\text { Kim (2014) } \\
\text { [31] }\end{array}$ & $\begin{array}{l}\text { F- } \\
\text { SWT }\end{array}$ & $\begin{array}{l}\text { Ultrasound } \\
\text { Needling }\end{array}$ & $\begin{array}{l}\text { Radiographic evaluations were } \\
\text { performed by standard shoulder } \\
\text { anteroposterior radiographs in } \\
\text { neutral, internal, and external } \\
\text { rotation together with axillary and } \\
\text { supraspinatus outlet views to } \\
\text { determine the size, morphology, } \\
\text { and location of the calcific } \\
\text { deposits. Resorption of the calcific } \\
\text { deposit was graded as none, } \\
\text { partial, or complete. }\end{array}$ & $\begin{array}{l}6 \text { weeks, } \\
3,6,12 \\
\text { months }\end{array}$ & $\begin{array}{l}\text { The mean Calcium deposits } \\
\text { diameter (mm) pre-SWT was } 11 \pm 1 \\
\text { (4.9-19.3), post-SWT was } 5.6 \pm 0.8 \text {. } \\
\text { In contrast, pre-US needling was } \\
14.8 \pm 1.7(6.6-31) \text {, post-US need- } \\
\text { ling was } 0.45 \pm 0.3 \text {. } \\
\text { In the SWT group, } 42.6 \% \text { had total } \\
\text { resorption, } 16.7 \% \text { had partial } \\
\text { resorption. In the US needling } \\
\text { group } 72.2 \% \text { had total resorption } \\
\text { and } 11.1 \% \text { had partial resorption. }\end{array}$ & $<0.05$ & $=0.001$ \\
\hline $\begin{array}{l}\text { Kransy (2005) } \\
\text { [32] }\end{array}$ & $\begin{array}{l}\text { F- } \\
\text { SWT }\end{array}$ & $\begin{array}{l}\text { Ultrasound } \\
\text { Needling } \\
\text { combined with } \\
\text { SWT }\end{array}$ & $\begin{array}{l}\text { Anteroposterior radiographs were } \\
\text { taken in internal and external } \\
\text { rotation together with axial and } \\
\text { supraspinatus-outlet views to de- } \\
\text { termine the size, morphology and } \\
\text { location of the calcific deposits. }\end{array}$ & $4.1 \pm 0.5$ & $\begin{array}{l}\text { In the SWT only group, } 13(32.5 \%) \\
\text { had total resorption, } 14(35 \%) \text { had } \\
\text { partial resorption. In the US } \\
\text { needling combined with SWT } \\
\text { group } 24(60 \%) \text { had total resorption } \\
\text { and } 10(25 \%) \text { had partial } \\
\text { resorption. }\end{array}$ & NS & $=0.024$ \\
\hline $\begin{array}{l}\text { Loew (1999) } \\
\text { [33] }\end{array}$ & $\begin{array}{l}\text { F- } \\
\text { SWT }\end{array}$ & $\begin{array}{l}\text { Low SWT and } \\
\text { control }\end{array}$ & $\begin{array}{l}\text { Radiographs included an } \\
\text { anteroposterior view in internal } \\
\text { and external rotation and a } \\
\text { supraspinatus outlet view. Effective } \\
\text { treatment was recorded when the } \\
\text { calcification had completely }\end{array}$ & $\begin{array}{l}3,6 \\
\text { months }\end{array}$ & $\begin{array}{l}\text { There was total resorption in } 4 / 20 \\
\text { in group } 1,11 / 20 \text { in group } 2,12 / \\
20 \text { in group } 3 \text {, in contrast to } 2 / 20 \\
\text { in the control group }\end{array}$ & $\begin{array}{l}\text { Group } \\
1= \\
0.37 \\
\text { Group } \\
2,3< \\
0.01\end{array}$ & NR \\
\hline
\end{tabular}

disappeared or showed obvious resorption with inhomogeneity and reduction in size

$\begin{array}{lll}\text { Lowe (1995) } & \text { F- } & \text { None } \\ \text { [34] } & \text { SWT }\end{array}$

Radiological assessment of the calcification was made in three
6,12 After 12 weeks, changes were seen NR weeks in 12 patients; 7 showed a total 
Table 2 Imaging outcome measures for rotator cuff calcifying tendinitis (Continued)

\begin{tabular}{|c|c|c|c|c|c|c|c|}
\hline \multirow[t]{3}{*}{ Author (year) } & \multirow{3}{*}{$\begin{array}{l}\text { SWT } \\
\text { type }\end{array}$} & \multirow[t]{3}{*}{ Comparator } & \multirow[t]{3}{*}{ Imaging outcome } & \multicolumn{4}{|l|}{ Follow-up } \\
\hline & & & & \multirow[t]{2}{*}{ Period } & \multirow{2}{*}{$\begin{array}{l}\text { Baseline }-F / U \\
\text { Mean } \pm S D\end{array}$} & \multicolumn{2}{|l|}{$\boldsymbol{P}$ value } \\
\hline & & & & & & $\begin{array}{l}\text { Within } \\
\text { group }\end{array}$ & $\begin{array}{l}\text { Between } \\
\text { group }\end{array}$ \\
\hline & & & different planes. & & resorption of the calcium deposits & & \\
\hline $\begin{array}{l}\text { Moretti } \\
\text { (2005) [35] }\end{array}$ & $\begin{array}{l}\text { F- } \\
\text { SWT }\end{array}$ & None & $\begin{array}{l}\text { Radiographs in anteroposterior of } \\
\text { shoulder, acromial outlet view and } \\
\text { sonography were evaluated to } \\
\text { study the type of calcium deposit } \\
\text { according to DePalma criteria }\end{array}$ & $\begin{array}{l}1,6 \\
\text { months }\end{array}$ & $\begin{array}{l}\text { 29/54 (54\%) had total resorption } \\
\text { and 19/54 (35) had partial } \\
\text { resorption after } 1 \text { month. These } \\
\text { findings appeared unvaried at } 6 \\
\text { months follow-up }\end{array}$ & NR & NA \\
\hline $\begin{array}{l}\text { Pan (2003) } \\
{[36]}\end{array}$ & $\begin{array}{l}\text { F- } \\
\text { SWT }\end{array}$ & TENS & $\begin{array}{l}\text { High-resolution ultrasonography } \\
\text { (HRUS) was used for imaging } \\
\text { measurements. The morphology of } \\
\text { calcific plaque of the shoulder on } \\
\text { HRUS was classified into } 4 \text { types: } \\
\text { (1) arc-shaped (echogenic arc with } \\
\text { clear shadowing), (2) fragmented } \\
\text { (at least } 2 \text { separated echogenic } \\
\text { plaques with or without shadow- } \\
\text { ing) or punctuated (tiny calcific } \\
\text { spots without shadowing), (3) } \\
\text { nodular (echogenic nodule with- } \\
\text { out shadowing), and (4) cystic } \\
\text { types (bold echogenic wall with } \\
\text { echo-free content) }\end{array}$ & $\begin{array}{l}2,4,12 \\
\text { weeks }\end{array}$ & $\begin{array}{l}\text { The mean of difference in Calcium } \\
\text { deposits diameter }(\mathrm{mm}) \text { in the } \\
\text { SWT group was } 4.39 \pm 3.76 \text { after } \\
12 \text { weeks. In contrast, the TENS } \\
\text { group was } 1.65 \pm 2.83 \text {. } \\
16 / 33(48.5 \%) \text { changed in the type } \\
\text { of calcification In the SWT group } \\
\text { while } 3 / 29(10.3) \text { in the TENS } \\
\text { group. }\end{array}$ & $<0.01$ & 0.002 \\
\hline $\begin{array}{l}\text { Pigozzi (2000) } \\
{[37]}\end{array}$ & $\begin{array}{l}\text { F- } \\
\text { SWT }\end{array}$ & None & $\begin{array}{l}\text { Radiological assessment of } \\
\text { anteroposterior, internal and } \\
\text { external rotation and trans-glenoid } \\
\text { projection was performed }\end{array}$ & 1 month & $\begin{array}{l}7 / 19(37 \%) \text { had reduction or } \\
\text { fragmentation of the calcium } \\
\text { deposit }\end{array}$ & NR & NA \\
\hline $\begin{array}{l}\text { Pleiner (2004) } \\
\text { [38] }\end{array}$ & $\begin{array}{l}\text { F- } \\
\text { SWT }\end{array}$ & Placebo & $\begin{array}{l}\text { Anteriorposterior, axial and outlet- } \\
\text { view images were used. Changes } \\
\text { calcifications were assessed using } \\
\text { the Gartner scale in which a score } \\
\text { of 1: indicates no change or a } \\
\text { worsening, a score of 2: a decrease } \\
\text { of at least } 50 \% \text { in the area and } \\
\text { density of the calcification, and a } \\
\text { score of 3: complete remission of } \\
\text { the calcification }\end{array}$ & $\begin{array}{l}3,7 \\
\text { months }\end{array}$ & $\begin{array}{l}6 / 31(19.4 \%) \text { had total resorption } \\
\text { and } 6 / 31(19.4 \%) \text { had partial } \\
\text { resorption in the SWT group, in } \\
\text { contrast to } 2 / 26(7.7 \%) \text { had total } \\
\text { resorption and } 2 / 26(7.7 \%) \text { had } \\
\text { partial resorption in the control } \\
\text { group after } 7 \text { months }\end{array}$ & NR & $=0.07$ \\
\hline $\begin{array}{l}\text { Rompe } \\
\text { (1995) [39] }\end{array}$ & $\begin{array}{l}\text { F- } \\
\text { SWT }\end{array}$ & None & $\begin{array}{l}\text { On radiographs, any sign of } \\
\text { disintegration was rated as success }\end{array}$ & $\begin{array}{l}6,24 \\
\text { weeks }\end{array}$ & $\begin{array}{l}4 / 40(10 \%) \text { had total resorption } \\
\text { and } 17 / 40(42.5 \%) \text { had partial } \\
\text { resorption after } 6 \text { weeks. } \\
\text { After } 24 \text { weeks, } 6 / 40(15 \%) \text { had } \\
\text { total resorption and } 19 / 40(47.5 \%) \\
\text { had partial resorption }\end{array}$ & NR & NA \\
\hline $\begin{array}{l}\text { Rompe } \\
\text { (2001) [40] }\end{array}$ & $\begin{array}{l}\text { F- } \\
\text { SWT }\end{array}$ & $\begin{array}{l}\text { Surgical } \\
\text { extirpation }\end{array}$ & $\begin{array}{l}\text { On the anteroposterior radiological } \\
\text { views, resorption was graded as } \\
\text { none, partial, or complete. }\end{array}$ & $\begin{array}{l}12 \\
\text { months }\end{array}$ & $\begin{array}{l}47 \% \text { had total resorption and } 33 \% \\
\text { had partial resorption in the SWT } \\
\text { group. In the surgical group, } 85 \% \\
\text { had total resorption and } 15 \% \text { had } \\
\text { partial resorption. There was no } \\
\text { significant difference regarding the } \\
\text { radio-morphologic features }\end{array}$ & $\mathrm{NR}$ & $<0.01$ \\
\hline $\begin{array}{l}\text { Sabeti- } \\
\text { Aschraf } \\
\text { (2005) [41] }\end{array}$ & $\begin{array}{l}\text { F- } \\
\text { SWT }\end{array}$ & $\begin{array}{l}\text { Navigation vs } \\
\text { feedback }\end{array}$ & $\begin{array}{l}\text { No change in the radiographs was } \\
\text { graded as } 4, \text { a } 3 \text { indicated slight } \\
\text { alteration of the calcium deposit, } \\
\text { reduction in deposit size and } \\
\text { radiographic density was graded } \\
\text { as } 2 \text {, and a } 1 \text { was given if the } \\
\text { calcium deposit was no longer } \\
\text { evident }\end{array}$ & $\begin{array}{l}3 \\
\text { months }\end{array}$ & $\begin{array}{l}6 / 25(24 \%) \text { had total resorption } \\
\text { and } 7 / 25(28 \%) \text { had extensive } \\
\text { resorption in the navigation group, } \\
\text { in contrast to } 1 / 25(4 \%) \text { had total } \\
\text { resorption and } 5 / 25(20 \%) \text { had } \\
\text { partial resorption in the feedback } \\
\text { group }\end{array}$ & $\mathrm{NR}$ & 0.041 \\
\hline $\begin{array}{l}\text { Tornese } \\
\text { (2011) [42] }\end{array}$ & $\begin{array}{l}\text { F- } \\
\text { SWT }\end{array}$ & $\begin{array}{l}\text { Neutral vs } \\
\text { hyperextended } \\
\text { internal } \\
\text { rotation arm }\end{array}$ & $\begin{array}{l}\text { Changes between pre- and post- } \\
\text { treatment radiographs were } \\
\text { graded as no resorption, partial re- } \\
\text { sorption and total or subtotal }\end{array}$ & $\begin{array}{l}3 \\
\text { months }\end{array}$ & $\begin{array}{l}12 / 18(66.7 \%) \text { had total resorption } \\
\text { in the hyperextended internal } \\
\text { rotation group, in contrast to } 6 / 17 \\
(35.3 \%) \text { in the neutral position }\end{array}$ & NR & $<0.05$ \\
\hline
\end{tabular}


Table 2 Imaging outcome measures for rotator cuff calcifying tendinitis (Continued)

\begin{tabular}{|c|c|c|c|c|c|c|c|}
\hline \multirow[t]{3}{*}{ Author (year) } & \multirow{3}{*}{$\begin{array}{l}\text { SWT } \\
\text { type }\end{array}$} & \multirow[t]{3}{*}{ Comparator } & \multirow[t]{3}{*}{ Imaging outcome } & \multicolumn{4}{|c|}{ Follow-up } \\
\hline & & & & \multirow[t]{2}{*}{ Period } & \multirow{2}{*}{$\begin{array}{l}\text { Baseline - F/U } \\
\text { Mean } \pm \text { SD }\end{array}$} & \multicolumn{2}{|l|}{$\boldsymbol{P}$ value } \\
\hline & & & & & & $\begin{array}{l}\text { Within } \\
\text { group }\end{array}$ & $\begin{array}{l}\text { Between } \\
\text { group }\end{array}$ \\
\hline & & position & $\begin{array}{l}\text { resorption (> } 80 \% \text { reduction in cal- } \\
\text { cified surface on anteroposterior } \\
\text { view) }\end{array}$ & & group & & \\
\hline
\end{tabular}

F-SWT focused SWT, $R$-SWT radial SWT, NR not reported, NS not significant, NA not applicable

Ultrasonography was the most common method for evaluating the imaging outcomes as reported in nine studies, followed by MRI in four studies. The change in plantar fascia thickness (PFT) was evaluated in eight studies as the most frequently reported imaging outcome (Table 4).

There was an overall reduction in PFT following SWT application (MD $0.92 \mathrm{~mm}$ (95\%CI 0.03, 1.81), $p=0.04$; Fig. 6) at 4 weeks [55], 6 weeks [54], 3 months [49, 53, 56] and 6 month follow-up $[47,51]$. Subgroup analysis showed greater reduction in PFT using radiological guidance (MD $1.31 \mathrm{~mm}$ (95\%CI 0.49, 2.13), $p=0.002$ ) versus no guidance (MD 0.47 $\mathrm{mm}(95 \% \mathrm{CI}-0.28,1.21), p=0.22)$ (Fig. 7). Baseline PFT was the only covariate to explain the variance related to the effect of SWT (Coeff. $-1.06 \mathrm{~mm}$ (95\%CI -1.59 to -0.53 ) $\mathrm{I}^{2}=78 \%$, Adj. $\left.\mathrm{R}^{2}=85.10 \%, p=0.004\right)$. No variables related to SWT treatment parameters were significant covariates. The reduction of PFT favored SWT compared with corticosteroid injection (MD $-0.3 \mathrm{~mm}$ (95\%CI -0.62-0.02), $p=0.07)[49,53,54]$ and over placebo control (MD - 0.9 $\mathrm{mm}(95 \% \mathrm{CI}-1.56$ to -0.24$), p=0.007)$ [56]. However, the effect of SWT on PFT was less compared to low-level laser therapy and therapeutic ultrasound (MD $0.43 \mathrm{~mm}$ (95\%CI 0.09, 0.78), $p=0.01$ ) [55] (Fig. 8).

The bone resorption effect of SWT on calcaneal spurs was evaluated in three studies (1 RCT) with a total of 461 participants. Chronic symptomatic heel pain ( $>3$ months) and radiological evidence of a calcaneal spur were the main inclusion criteria. Radial SWT was used in two studies and one study employed focused SWT. None of the studies utilized radiological guidance and only one study used anesthesia [50]. The range of delivered shocks was
1500-2000 shocks carried in 1-5 sessions with 7-30 days interval between each session (Table 3 ).

Morphological changes in calcaneal spurs were evaluated using plain radiographs in two studies, while one study [46] measured bone mineral density (BMD) and bone mineral content (BMC) using DEXA scanning. None of the studies reported calcaneal spur fragmentation or significant reduction of the spur dimensions $[50,57]$. However, both BMD and BMC demonstrated statistically significant improvement following focused SWT after a 12 week follow-up period, indicating an osteogenic effect [46] (Table 4).

\section{Osteonecrosis of the femoral head}

The effects of SWT on osteonecrosis of the femoral head (ONFH) were evaluated in 12 studies (4 RCTs) published between 2001 and 2018. These studies included 325 (404 hips) participants with stage I-III ONFH according to the Association Research Circulation Osseous (ARCO) classification as the main inclusion criterion, except for one study [59] that included ARCO stage I only. The main exclusion criteria were late ARCO stages, infection, advanced arthritis, neoplastic disease or blood coagulation disorders.

All of the included studies employed focused SWT with a mean EFD of $0.57 \pm 0.06(0.47-0.62) \mathrm{mJ} / \mathrm{mm}^{2}$ and a mean of $4867.86 \pm 1469.64$ (2000-6000) shocks. All of the included studies delivered the treatment over one session, except Vulpiani et al. [60] who provided treatment over four sessions and D'Agostino et al. [59] over two sessions (2-3 days interval). Anesthesia was used in eight studies and radiological guidance was implemented in nine studies to accurately target the site of lesion (Table 5).

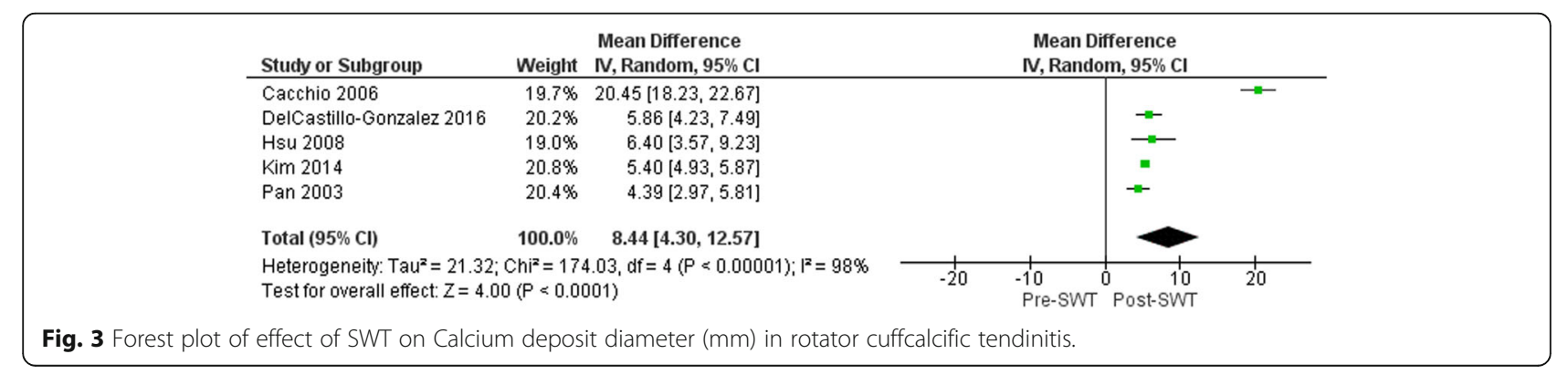




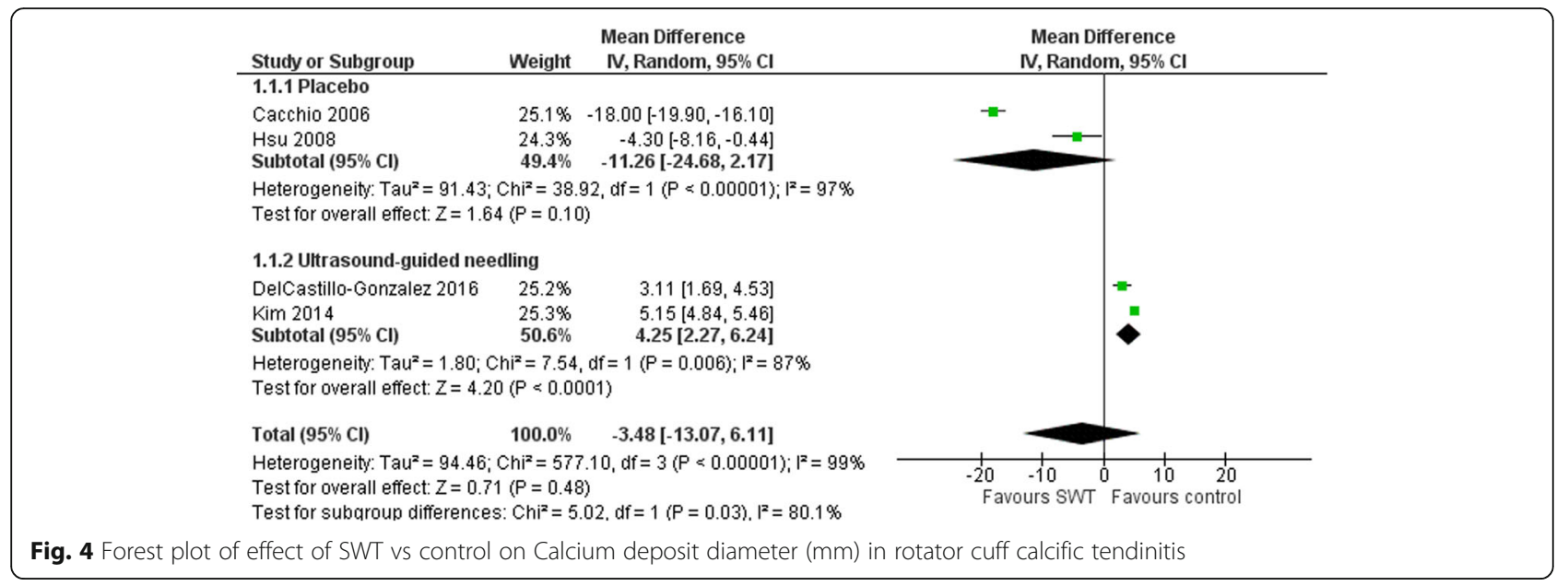

Imaging changes of ONFH were measured utilizing MRI in addition to radiography in all included studies to evaluate the lesion size, femoral head congruency, presence of a crescent sign, BME and degenerative changes of the hip joint. The percentage of change in the osteonecrosis lesion size was reported in eight studies (Table 6).

The size of the lesion (\%) showed modest reduction following SWT application with marginal statistical significance (MD 4.84\% (95\%CI -0.06-9.75), $p=0.05$; Fig. 9) at 6 months [65], 12 months [70], 2 years [61, 63, 66-69] and 3 years [62] follow-up. Baseline lesion size was the only covariate to explain the variance related to the effect of SWT (Coeff. 0.87\% (95\%CI 0.48, 1.26) $\mathrm{I}^{2}=6.2 \%$, Adj. $R^{2}=93.77 \%, p=0.001$ ). No variables related to SWT treatment parameters were significant covariates.
The reduction in the lesion size generally favored SWT compared to other interventions such as core decompression [66, 68], cocktail therapy [63] and SWT combined with alendronate [69] with an overall MD -8.50\% (95\%CI -16.40 to -0.59 ), $p=0.04$; Fig. 10.

\section{Miscellaneous conditions}

The remaining musculoskeletal conditions evaluated with imaging measures following SWT were fracture nonunion [71-74], lateral epicondylitis [75, 76], knee osteoarthritis related BME [77, 78], Achilles tendinopathy [79], post-traumatic myositis ossificans [80], arthroscopic rotator cuff repair [81] and Kienbock's disease [82]. Description of these studies is provided in Additional file 3.

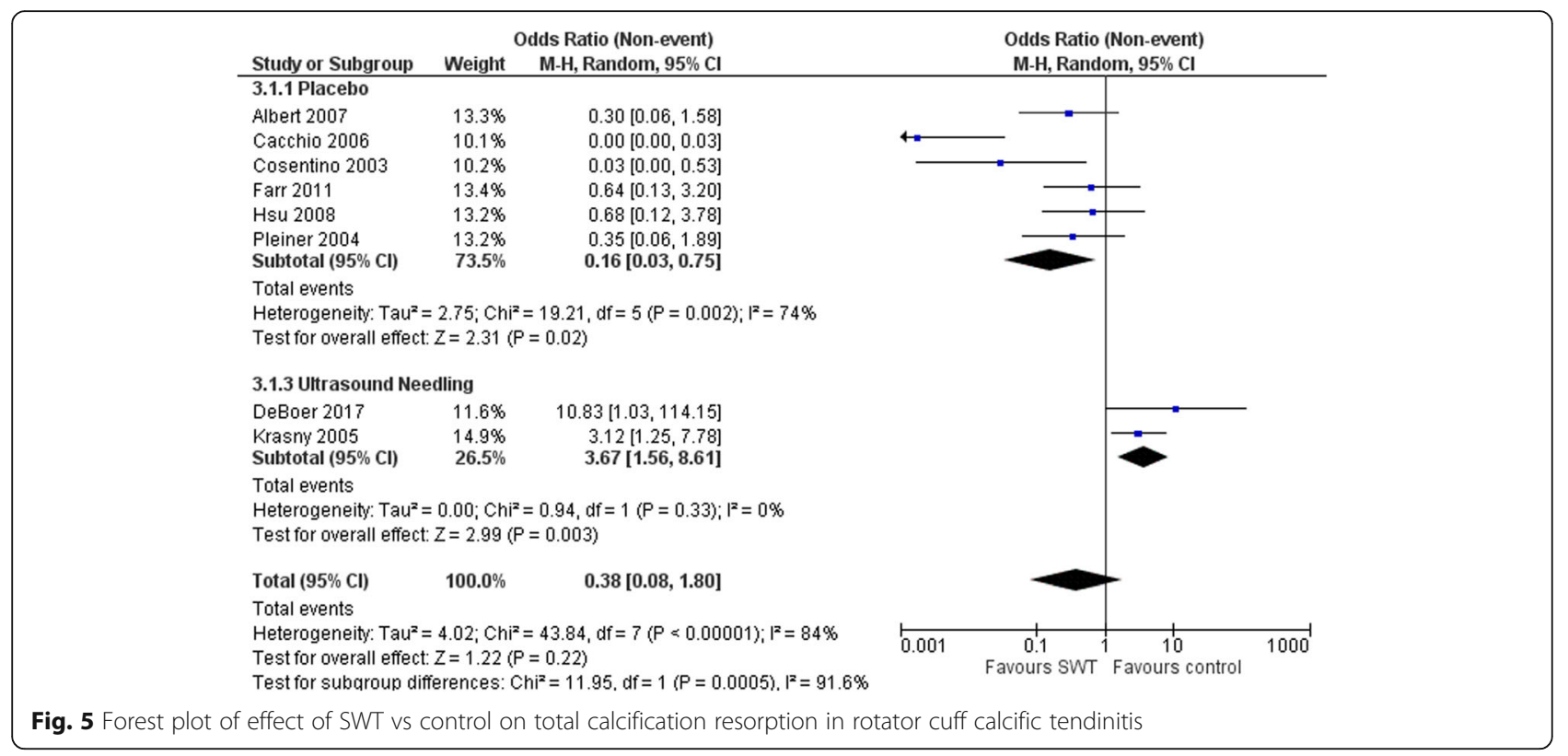


Table 3 Characteristics of studies and intervention details for plantar fasciitis and heel spurs

\begin{tabular}{|c|c|c|c|c|c|c|c|c|c|c|}
\hline Author & $\begin{array}{l}\text { Study } \\
\text { design }\end{array}$ & Condition & $N$ & $\begin{array}{l}\text { Mean age } \pm \\
\text { SD or } \\
\text { (range) }\end{array}$ & $\begin{array}{l}\text { Mean } \\
\text { symptoms } \\
\text { duration } \pm \\
\text { SD or } \\
\text { (range), } \\
\text { months }\end{array}$ & $\begin{array}{l}\text { Area of SWT } \\
\text { application }\end{array}$ & $\begin{array}{l}\text { Dosage in } \\
\text { impulses*EFD } \\
\left(\mathrm{mJ} / \mathrm{mm}^{2}\right) / \mathrm{bar}\end{array}$ & $\begin{array}{l}\text { No. of } \\
\text { sessions }\end{array}$ & $\begin{array}{l}\text { Interval } \\
\text { between } \\
\text { sessions }\end{array}$ & $\begin{array}{l}\text { Co- } \\
\text { intervention, } \\
\text { anesthesia }\end{array}$ \\
\hline $\begin{array}{l}\text { Bicer (2018) } \\
\text { [44] }\end{array}$ & $\begin{array}{l}\text { Prospective } \\
\text { open } \\
\text { design }\end{array}$ & $\begin{array}{l}\text { Plantar } \\
\text { fasciitis }\end{array}$ & 30 & $45.23 \pm 8.57$ & $>6$ weeks & NR & $2500 * 2-3$ bars & 3 & 1 week & None \\
\hline $\begin{array}{l}\text { Chew (2013) } \\
\text { [45] }\end{array}$ & $\mathrm{RCT}$ & $\begin{array}{l}\text { Plantar } \\
\text { fasciitis }\end{array}$ & 19 & $\begin{array}{l}\text { median and } \\
\text { interquartile } \\
\text { range: } 45 \\
\text { (37-53) }\end{array}$ & $\begin{array}{l}\text { median and } \\
\text { interquartile } \\
\text { range: } 18 \\
\text { (7-24) }\end{array}$ & $\begin{array}{l}\text { SWT delivered under } \\
\text { ultrasound guidance } \\
\text { to the painful and } \\
\text { thickened region of } \\
\text { the plantar fascia at } \\
\text { the medial calcaneal } \\
\text { tubercle. The patient } \\
\text { was positioned } \\
\text { prone with the feet } \\
\text { over the end of the } \\
\text { table }\end{array}$ & $2000 * 0.42$ & 2 & 1 week & $\begin{array}{l}\text { Home } \\
\text { exercise } \\
\text { program, } \\
\text { none }\end{array}$ \\
\hline $\begin{array}{l}\text { Daniel-Lucian } \\
\text { (2013) [43] }\end{array}$ & $\begin{array}{l}\text { Prospective } \\
\text { open } \\
\text { design }\end{array}$ & $\begin{array}{l}\text { Plantar } \\
\text { fasciitis }\end{array}$ & 17 & NR & $>3$ & $\begin{array}{l}\text { SWT was applied } \\
\text { over the calcaneal } \\
\text { plantar side, } \\
\text { aponeurosis insertion } \\
\text { and plantar } \\
\text { aponeurosis }\end{array}$ & $\begin{array}{l}4000 \\
E F D \text { is NR }\end{array}$ & 8 & $\begin{array}{l}\text { Twice a } \\
\text { week }\end{array}$ & None \\
\hline $\begin{array}{l}\text { Gerdesmeyer } \\
\text { (2015) [46] }\end{array}$ & $\begin{array}{l}\text { prospective } \\
\text { intra- } \\
\text { individual } \\
\text { controlled }\end{array}$ & Heel spur & 45 & $\begin{array}{l}53(28.6- \\
80.1)\end{array}$ & $>6$ & $\begin{array}{l}\text { SWT localization of } \\
\text { the most painful } \\
\text { area was achieved } \\
\text { with biofeedback } \\
\text { mechanism and } \\
\text { radiologically } \\
\text { controlled. SWT was } \\
\text { placed at the origin } \\
\text { of the fascia and } \\
\text { reached the central } \\
\text { calcaneus. }\end{array}$ & $2000 * 0.32$ & 2 & 2 weeks & None \\
\hline $\begin{array}{l}\text { Hammer } \\
\text { (2005) [47] }\end{array}$ & $\begin{array}{l}\text { prospective } \\
\text { intra- } \\
\text { individual } \\
\text { controlled }\end{array}$ & $\begin{array}{l}\text { Plantar } \\
\text { fasciitis }\end{array}$ & 22 & $51.6(24-79)$ & $8.8(6-12)$ & NR & $3000 * 0.2$ & 3 & 1 week & None \\
\hline $\begin{array}{l}\text { Hocaoglu } \\
\text { (2017) [48] }\end{array}$ & $\mathrm{RCT}$ & $\begin{array}{l}\text { Plantar } \\
\text { fasciitis }\end{array}$ & 36 & $50.22 \pm 8.29$ & $8(6-24)$ & $\begin{array}{l}\text { Patients were in the } \\
\text { prone position. SWT } \\
\text { was applied on } \\
\text { plantar fascia } \\
\text { insertion of the } \\
\text { calcaneus }\end{array}$ & $2000 * 0.16$ & 3 & 1 week & None \\
\hline $\begin{array}{l}\text { Lai (2018) } \\
\text { [49] }\end{array}$ & $\mathrm{RCT}$ & $\begin{array}{l}\text { Plantar } \\
\text { fasciitis }\end{array}$ & 47 & $54.53 \pm 8.62$ & $7.94 \pm 2.92$ & NR & $1500 * 029$ & 2 & 2 weeks & None \\
\hline $\begin{array}{l}\text { Lee (2003) } \\
\text { [50] }\end{array}$ & $\mathrm{RCT}$ & $\begin{array}{l}\text { Calcaneal } \\
\text { bone } \\
\text { spurs }\end{array}$ & 308 & NR & $>6$ & $\begin{array}{l}\text { SWT was applied } \\
\text { from the plantar } \\
\text { surface over a } 2 \mathrm{~cm} \\
\text { circular area around } \\
\text { the predetermined } \\
\text { point of maximal } \\
\text { tenderness at the } \\
\text { plantar anthesis }\end{array}$ & $1500 * 0.22$ & 1 or 2 & 3 months & None, yes \\
\hline $\begin{array}{l}\text { Maki (2017) } \\
\text { [51] }\end{array}$ & $\begin{array}{l}\text { Prospective } \\
\text { open } \\
\text { design }\end{array}$ & $\begin{array}{l}\text { Plantar } \\
\text { fasciitis }\end{array}$ & 23 & $55.3(16-81)$ & $26.9(4-300)$ & $\begin{array}{l}\text { SWT was applied at } \\
\text { the plantar fascia } \\
\text { attachment under } \\
\text { ultrasonic guidance } \\
\text { from the medial } \\
\text { calcaneus. A second } \\
\text { treatment was } \\
\text { performed if }\end{array}$ & $3800 * 0.36$ & 1 or 2 & 3 months & None \\
\hline
\end{tabular}


Table 3 Characteristics of studies and intervention details for plantar fasciitis and heel spurs (Continued)

\begin{tabular}{|c|c|c|c|c|c|c|c|c|c|c|}
\hline Author & $\begin{array}{l}\text { Study } \\
\text { design }\end{array}$ & Condition & N & $\begin{array}{l}\text { Mean age } \pm \\
\text { SD or } \\
\text { (range) }\end{array}$ & $\begin{array}{l}\text { Mean } \\
\text { symptoms } \\
\text { duration } \pm \\
\text { SD or } \\
\text { (range), } \\
\text { months }\end{array}$ & $\begin{array}{l}\text { Area of SWT } \\
\text { application }\end{array}$ & $\begin{array}{l}\text { Dosage in } \\
\text { impulses*EFD } \\
\left(\mathrm{mJ} / \mathrm{mm}^{2}\right) / \mathrm{bar}\end{array}$ & $\begin{array}{l}\text { No. of } \\
\text { sessions }\end{array}$ & $\begin{array}{l}\text { Interval } \\
\text { between } \\
\text { sessions }\end{array}$ & $\begin{array}{l}\text { Co- } \\
\text { intervention, } \\
\text { anesthesia }\end{array}$ \\
\hline & & & & & & $\begin{array}{l}\text { symptoms persisted } \\
\text { at } 3 \text { months }\end{array}$ & & & & \\
\hline $\begin{array}{l}\text { Moretti } \\
\text { (2006) [52] }\end{array}$ & $\begin{array}{l}\text { Prospective } \\
\text { open } \\
\text { design }\end{array}$ & $\begin{array}{l}\text { Plantar } \\
\text { fasciitis }\end{array}$ & 54 & $35.2(30-42)$ & $>6$ & $\begin{array}{l}\text { SWT was applied at } \\
\text { the medial tubercle } \\
\text { of the calcaneus, at } \\
\text { the proximal } \\
\text { insertion of the } \\
\text { plantar fascia or the } \\
\text { calcaneal spur, } \\
\text { around the point of } \\
\text { medial tenderness }\end{array}$ & $2000 * 0.04$ & 4 & 1 week & None \\
\hline $\begin{array}{l}\text { Saber (2012) } \\
\text { [53] }\end{array}$ & $\mathrm{RCT}$ & $\begin{array}{l}\text { Plantar } \\
\text { fasciitis }\end{array}$ & 30 & $34.27 \pm 7.19$ & $>6$ & $\begin{array}{l}\text { SWT was applied in } \\
\text { prone position over } \\
\text { the area of maximal } \\
\text { tenderness and } \\
\text { finding by } \\
\text { ultrasonography }\end{array}$ & $\begin{array}{l}1000- \\
1500^{*} 0.28\end{array}$ & 2 & 2 weeks & None, yes \\
\hline $\begin{array}{l}\text { Sorrentino } \\
\text { (2008) [54] }\end{array}$ & RCT & $\begin{array}{l}\text { Plantar } \\
\text { fasciitis }\end{array}$ & 30 & $\begin{array}{l}\text { Total } \\
\text { sample }=34 \\
\text { women } \\
(56 \pm 2.4) \\
\text { and } 26 \text { men } \\
(52 \pm 3.7)\end{array}$ & 4 & $\begin{array}{l}\text { SWT was applied } \\
\text { under } \\
\text { ultrasonography } \\
\text { guidance to locate } \\
\text { the calcaneal } \\
\text { insertion of the } \\
\text { plantar fascia }\end{array}$ & $2000 * 0.03$ & 4 & 1 week & None \\
\hline $\begin{array}{l}\text { Ulusoy (2017) } \\
\text { [55] }\end{array}$ & $\mathrm{RCT}$ & $\begin{array}{l}\text { Plantar } \\
\text { fasciitis }\end{array}$ & 19 & $54.45 \pm 6.9$ & $27 \pm 29.79$ & $\begin{array}{l}\text { SWT was applied in } \\
\text { the prone position } \\
\text { into the areas of the } \\
\text { painful heel, } \\
\text { insertion of plantar } \\
\text { fascia on the medial } \\
\text { calcaneal area, and } \\
\text { myofascial junction } \\
\text { at the dorsum of the } \\
\text { heel }\end{array}$ & $2000 * 2.5$-bar & 3 & 1 week & $\begin{array}{l}\text { Continue } \\
\text { previous } \\
\text { exercise } \\
\text { program, no }\end{array}$ \\
\hline $\begin{array}{l}\text { Vahdatpour } \\
\text { (2012) [56] }\end{array}$ & RCT & $\begin{array}{l}\text { Plantar } \\
\text { fasciitis }\end{array}$ & 20 & $50.6 \pm 10$ & $>3$ & $\begin{array}{l}\text { SWT was targeted to } \\
\text { the maximum local } \\
\text { tenderness area }\end{array}$ & $\begin{array}{l}2000 \\
\text { focused }+ \\
2000 \\
\text { radial*0.2 }\end{array}$ & 3 & 1 week & $\begin{array}{l}\text { Exercise, } \\
\text { NSAIDs, and } \\
\text { heel pad for } \\
\text { both groups, } \\
\text { no }\end{array}$ \\
\hline $\begin{array}{l}\text { Yalcin (2012) } \\
\text { [57] }\end{array}$ & $\begin{array}{l}\text { Prospective } \\
\text { open } \\
\text { design }\end{array}$ & Heel spur & 108 & $50.2 \pm 11.3$ & $27.4 \pm 32.8$ & $\begin{array}{l}\text { SWT was applied in } \\
\text { prone position to } \\
\text { the marked tender } \\
\text { spot }\end{array}$ & $\begin{array}{l}2000^{*} 0.4(4 \\
\text { bar) }\end{array}$ & 5 & 1 week & None \\
\hline $\begin{array}{l}\text { Zhu (2005) } \\
\text { [58] }\end{array}$ & $\begin{array}{l}\text { Prospective } \\
\text { open } \\
\text { design }\end{array}$ & $\begin{array}{l}\text { Plantar } \\
\text { fasciitis }\end{array}$ & $\begin{array}{l}12 \\
(18 \\
\mathrm{ft})\end{array}$ & $49.9(33-63)$ & $>6$ & $\begin{array}{l}\text { SWT was applied to } \\
\text { the most painful } \\
\text { point }(2-3 \mathrm{~cm} \\
\text { diameter) on the } \\
\text { heel }\end{array}$ & $1500 * 18 \mathrm{kv}$ & 1 & NA & None, yes \\
\hline
\end{tabular}

\section{Discussion}

The aim of the current systematic review and meta-analysis was to evaluate changes in the morphology of musculoskeletal structures as measured by imaging following a SWT (focused and radial) intervention. Overall, there was a tendency for SWT to demonstrate morphological changes among most of the included musculoskeletal conditions based on different quantitative imaging methods. These tissue changes tended to favor SWT over placebo or other comparators except for rotator cuff calcific tendinitis that favored ultrasound guided needling over SWT. Interestingly, SWT type (radial and focused) and the therapeutic dosage parameters did not appear to have a significant influence on the evaluated imaging outcomes according to our subgroup and meta- 
Table 4 Imaging outcome measures for plantar fasciitis and heel spurs

\begin{tabular}{|c|c|c|c|c|c|c|c|}
\hline \multirow[t]{3}{*}{ Author (year) } & \multirow{3}{*}{$\begin{array}{l}\text { SWT } \\
\text { type }\end{array}$} & \multirow[t]{3}{*}{ Comparator } & \multirow[t]{3}{*}{ Imaging outcome } & \multicolumn{4}{|c|}{ Follow-up } \\
\hline & & & & \multirow[t]{2}{*}{ Period } & \multirow{2}{*}{$\begin{array}{l}\text { Baseline - F/U } \\
\text { Mean } \pm \text { SD }\end{array}$} & \multicolumn{2}{|l|}{$\boldsymbol{P}$ value } \\
\hline & & & & & & $\begin{array}{l}\text { Within } \\
\text { group }\end{array}$ & $\begin{array}{l}\text { Between } \\
\text { group }\end{array}$ \\
\hline $\begin{array}{l}\text { Bicer (2018) } \\
\text { [44] }\end{array}$ & $\begin{array}{l}\text { R- } \\
\text { SWT }\end{array}$ & None & $\begin{array}{l}\text { MRI was used to assess changes in } \\
\text { the soft tissue and BME, plantar fascia } \\
\text { thickness (PFT) and the presence of } \\
\text { heel spurs. MRls were scored semi- } \\
\text { quantitatively. PFT was measured } 1 \mathrm{~cm} \\
\text { from the insertion and thickness }>3 \\
\text { mm was considered abnormal }\end{array}$ & $\begin{array}{l}3 \\
\text { months }\end{array}$ & $\begin{array}{l}\text { 12/23 (52.1\%) showed } \\
\text { improvement in PFT. } 21 / 30 \text { (70\%) } \\
\text { and 10/19(52.6\%) had } \\
\text { improvement in soft tissue and } \\
\text { BME respectively. No significant } \\
\text { change in heel spur }\end{array}$ & $<0.05$ & NA \\
\hline $\begin{array}{l}\text { Chew (2013) } \\
\text { [45] }\end{array}$ & $\begin{array}{l}\text { F- } \\
\text { SWT }\end{array}$ & $\begin{array}{l}\text { Autologous } \\
\text { Conditioned } \\
\text { Plasma (ACP) } \\
\text { and } \\
\text { conventional }\end{array}$ & $\begin{array}{l}\text { Ultrasonography of plantar fascia (PF) } \\
\text { was performed to manually measure } \\
\text { the point of maximal proximal PFT at } \\
\text { the medial calcaneal tubercle insertion } \\
\text { site. }\end{array}$ & $\begin{array}{l}1,3,6 \\
\text { months }\end{array}$ & $\begin{array}{l}\text { The median PFT improvement in } \\
\text { the ACP group at the } 6 \text {-month } \\
\text { follow-up was } 1.3 \mathrm{~mm} \text { compared } \\
\text { with the SWT and conventional } \\
\text { treatment groups, which both } \\
\text { showed improvements of } 0.6 \mathrm{~mm} \text {. }\end{array}$ & NR & $\begin{array}{l}\text { SWT vs } \\
\text { conventional } \\
\text { treatment }= \\
0.934 \\
\text { ACP vs } \\
\text { SWT }=0.027\end{array}$ \\
\hline $\begin{array}{l}\text { Daniel-Lucian } \\
\text { (2013) [43] }\end{array}$ & NR & None & $\begin{array}{l}\text { Ultrasonography was used to measure } \\
\text { PFT }\end{array}$ & $\begin{array}{l}3 \\
\text { months }\end{array}$ & $\begin{array}{l}\text { The mean PFT decreased from } \\
5.84 \text { to } 5.21 \text { in the females, and } \\
\text { from } 5.87 \text { to } 5.14 \text { in the male } \\
\text { subjects }\end{array}$ & NR & NR \\
\hline $\begin{array}{l}\text { Gerdesmeyer } \\
\text { (2015) [46] }\end{array}$ & $\begin{array}{l}\text { F- } \\
\text { SWT }\end{array}$ & None & $\begin{array}{l}\text { Measurements of bone mass density } \\
\text { (BMD) and bone mass concentration } \\
\text { (BMC) were performed with a Lunar } \\
\text { DEXA. The square-shaped analysis field } \\
\text { (Region of Interest, ROI) was placed in } \\
\text { the cancellous part of the calcanei } \\
\text { and BMD and BMC were measured. }\end{array}$ & $\begin{array}{l}6,12 \\
\text { weeks }\end{array}$ & $\begin{array}{l}\text { The mean BMD }(\mathrm{g} / \mathrm{cm} 2) \text { values } \\
\text { changed from } 0.5 \pm 0.1 \text { to } 0.557 \pm \\
0.1 \text { in the SWT group and from } \\
0.54 \pm 0.1 \text { to } 0.52 \pm 0.09 \text { in the } \\
\text { control group after } 12 \text { weeks. } \\
\text { The mean BMC }(\mathrm{g}) \text { values } \\
\text { changed from } 2.03 \pm 0.38 \text { to } \\
2.22 \pm 0.38 \text { in the SWT group and } \\
\text { from } 2.16 \pm 0.4 \text { to } 2.08 \pm 0.36 \text { in } \\
\text { the control group after } 12 \text { weeks }\end{array}$ & 0.001 & $<0.01$ \\
\hline $\begin{array}{l}\text { Hammer } \\
(2005)[47]\end{array}$ & $\begin{array}{l}\text { F- } \\
\text { SWT }\end{array}$ & None & $\begin{array}{l}\text { The PFT was measured about } 2 \mathrm{~cm} \\
\text { distal of the medial calcaneal } \\
\text { tuberosity using ultrasonography }\end{array}$ & $\begin{array}{l}6,12,24 \\
\text { weeks }\end{array}$ & $\begin{array}{l}\text { The mean PFT in } 16 \text { subjects } \\
\text { changed from } 5.2 \pm 1.5 \text { to } 4.4 \pm 1 \\
\text { after } 6 \text { months. There was no } \\
\text { significant change of PFT on the } \\
\text { control side }\end{array}$ & $<0.05$ & $<0.05$ \\
\hline $\begin{array}{l}\text { Hocaoglu } \\
\text { (2017) [48] }\end{array}$ & $\begin{array}{l}\text { R- } \\
\text { SWT }\end{array}$ & $\begin{array}{l}\text { Ultrasound- } \\
\text { guided local } \\
\text { corticosteroid } \\
\text { injection }\end{array}$ & $\begin{array}{l}\text { PFT and its echogenicity were } \\
\text { examined through ultrasonography. A } \\
\text { linear probe was positioned } \\
\text { longitudinally over the medial } \\
\text { tubercle of the calcaneus. PFT was } \\
\text { measured at the proximal point of } \\
\text { insertion of the fascia into the } \\
\text { calcaneal tubercle. A PFT of } 4 \mathrm{~mm} \text { was } \\
\text { considered evidence of fasciitis. }\end{array}$ & $\begin{array}{l}1,3,6 \\
\text { months }\end{array}$ & $\begin{array}{l}\text { PFT was found to be significantly } \\
\text { reduced in both groups at all } \\
\text { measurement endpoints } \\
\text { compared with baseline with no } \\
\text { significant differences between } \\
\text { groups }\end{array}$ & $<0.01$ & $>0.05$ \\
\hline $\begin{array}{l}\text { Lai (2018) } \\
{[49]}\end{array}$ & $\begin{array}{l}\text { F- } \\
\text { SWT }\end{array}$ & $\begin{array}{l}\text { Corticosteroid } \\
\text { injection }\end{array}$ & $\begin{array}{l}\text { PFT was measured at the PF insertion } \\
5 \mathrm{~mm} \text { distal to calcaneus tuberosity } \\
\text { using ultrasonography. }\end{array}$ & $\begin{array}{l}1,3 \\
\text { months }\end{array}$ & $\begin{array}{l}\text { At } 4 \text { th week, the mean PFT } \\
\text { changed in SWT group from } \\
0.37 \pm 0.07 \text { to } 0.46 \pm 0.08 \mathrm{~cm} \text {, and } \\
\text { in the CSI group from } 0.38 \pm 0.06 \\
\text { to } 0.43 \pm 0.09 \mathrm{~cm} \\
\text { At the } 12 \text { th week, the mean PFT } \\
\text { changed in the SWT group from } \\
0.37 \pm 0.07 \text { to } 0.38 \pm 0.07 \mathrm{~cm} \text {, and } \\
\text { the CSI group from } 0.38 \pm 0.06 \text { to } \\
0.39 \pm 0.07 \mathrm{~cm}\end{array}$ & NR & $\begin{array}{l}\text { At } 4 \text { th week } \\
=0.048 \\
\text { At } 12 \text { th week } \\
=0.326\end{array}$ \\
\hline $\begin{array}{l}\text { Lee (2003) } \\
{[50]}\end{array}$ & $\begin{array}{l}\text { R- } \\
\text { SWT }\end{array}$ & Placebo & $\begin{array}{l}\text { Axial, lateral, and oblique radiographs } \\
\text { of the calcaneus were performed to } \\
\text { examine the presence of any osseous } \\
\text { abnormalities of the calcaneus or for } \\
\text { the presence of inferior calcaneal } \\
\text { spurs }\end{array}$ & $\begin{array}{l}3,12 \\
\text { months }\end{array}$ & $\begin{array}{l}\text { 205/308 (67\%) in the EWST group } \\
\text { had an inferior calcaneal spur. In } \\
\text { the sham treatment group, } 78 / 127 \\
(61 \%) \text { had a spur. } \\
\text { No patient treated with SWT had } \\
\text { subsequent fragmentation or } \\
\text { disappearance of the heel spur at } \\
3 \text { or } 12 \text { months. Similarly, no } \\
\text { patient had evidence of reactive }\end{array}$ & NR & NR \\
\hline
\end{tabular}


Table 4 Imaging outcome measures for plantar fasciitis and heel spurs (Continued)

\begin{tabular}{|c|c|c|c|c|c|c|c|}
\hline \multirow[t]{3}{*}{ Author (year) } & \multirow{3}{*}{$\begin{array}{l}\text { SWT } \\
\text { type }\end{array}$} & \multirow[t]{3}{*}{ Comparator } & \multirow[t]{3}{*}{ Imaging outcome } & \multicolumn{4}{|c|}{ Follow-up } \\
\hline & & & & \multirow[t]{2}{*}{ Period } & \multirow{2}{*}{$\begin{array}{l}\text { Baseline }-F / U \\
\text { Mean } \pm S D\end{array}$} & \multicolumn{2}{|l|}{$\boldsymbol{P}$ value } \\
\hline & & & & & & $\begin{array}{l}\text { Within } \\
\text { group }\end{array}$ & $\begin{array}{l}\text { Between } \\
\text { group }\end{array}$ \\
\hline & & & & & $\begin{array}{l}\text { new bone formation in or around } \\
\text { the spur, nor apparent elongation } \\
\text { of the spur }\end{array}$ & & \\
\hline $\begin{array}{l}\text { Maki (2017) } \\
\text { [51] }\end{array}$ & $\begin{array}{l}\text { F- } \\
\text { SWT }\end{array}$ & None & $\begin{array}{l}\text { On MRI, } 4 \text { items were examined: PFT, } \\
\text { high-signal intensity area (HSIA) inside } \\
\text { the PF, edema around the PF, and } \\
\text { BME of the calcaneus. For the PFT, the } \\
\text { maximum diameter of the PF at the } \\
\text { calcaneal attachment was measured } \\
\text { on T1-weighted coronal images. }\end{array}$ & 6 & $\begin{array}{l}\text { The mean PFT changed from } \\
4.4 \pm 1.6 \text { to } 4.6 \pm 1.8 \text { after } 6 \\
\text { months. } \\
\text { The numbers of feet showing } \\
\text { HSIA inside the PF changed from } \\
15 \text { to } 6 \text {, in edema around the PF } \\
\text { from } 16 \text { to } 2 \text {, and in BME of the } \\
\text { calcaneus from } 11 \text { to } 4 \text {. }\end{array}$ & $>0.05$ & NA \\
\hline $\begin{array}{l}\text { Moretti } \\
\text { (2006) [52] }\end{array}$ & $\begin{array}{l}\text { F- } \\
\text { SWT }\end{array}$ & None & $\begin{array}{l}\text { A lateral weight-bearing X-ray of the } \\
\text { foot and ultrasound evaluation was } \\
\text { performed. }\end{array}$ & $\begin{array}{l}45 \\
\text { days, } 6, \\
24 \\
\text { months }\end{array}$ & $\begin{array}{l}\text { There was no heel spur } \\
\text { fragmentation observed. The } \\
\text { ultrasound evaluation at } 24 \\
\text { months showed a complete } \\
\text { disappearance of the inflammatory } \\
\text { signs in } 33(61 \%) \text { patients. }\end{array}$ & NR & NA \\
\hline $\begin{array}{l}\text { Saber (2012) } \\
\text { [53] }\end{array}$ & $\begin{array}{l}\text { F- } \\
\text { SWT }\end{array}$ & $\begin{array}{l}\text { Ultrasound- } \\
\text { guided local } \\
\text { corticosteroid } \\
\text { injection }\end{array}$ & $\begin{array}{l}\text { PFT was measured at the thickest } \\
\text { portion from the base of the medial } \\
\text { calcaneal tubercle where a bright } \\
\text { echogenic line was easily visible using } \\
\text { ultrasonography }\end{array}$ & $\begin{array}{l}20(12- \\
24) \\
\text { weeks }\end{array}$ & $\begin{array}{l}\text { The mean PFT in the SWT group } \\
\text { changed from } 5.93 \pm 0.54 \text { to } \\
3.37 \pm 0.42 \text {, and in the ultrasound } \\
\text { guided injection group from } \\
5.96 \pm 0.46 \text { to } 3.54 \pm 0.31\end{array}$ & $<0.01$ & $=0.079$ \\
\hline $\begin{array}{l}\text { Sorrentino } \\
\text { (2008) [54] }\end{array}$ & $\begin{array}{l}\text { F- } \\
\text { SWT }\end{array}$ & $\begin{array}{l}\text { Corticosteroid } \\
\text { injection }\end{array}$ & $\begin{array}{l}\text { Ultrasonography was performed in } \\
\text { prone position with ankles dorsiflexed. } \\
\text { The focus was adjusted to the depth } \\
\text { of the PF. The sonographic diagnosis } \\
\text { established based on: 1) fascial } \\
\text { thickening }>5 \mathrm{~mm}, 2 \text { ) biconvex } \\
\text { morphology and 3) abnormal fascial } \\
\text { echostructure, specifically } \\
\text { hypoechogenicity, heterogeneity and } \\
\text { ill-defined margins. PFT was measured } \\
1 \mathrm{~cm} \text { from the calcaneal insertion with } \\
\text { electronic calipers }\end{array}$ & weeks & $\begin{array}{l}\text { In the SWT group, PFT with } \\
\text { perifascial edema was reduced to } \\
4.6 \pm 0.6 \mathrm{~mm} \text { and up to } 4 \pm 0.3 \mathrm{~mm} \\
\text { among PFT without perifascial } \\
\text { edema. } \\
\text { In the corticosteroid } \\
\text { Injection, PFT with perifascial } \\
\text { edema was reduced to } \\
4.3 \pm 0.4 \mathrm{~mm} \text { and up to } 4.6 \pm 0.4 \\
\mathrm{~mm} \text { among PFT without } \\
\text { perifascial edema }\end{array}$ & NR & NR \\
\hline $\begin{array}{l}\text { Ulusoy (2017) } \\
\text { [55] }\end{array}$ & $\begin{array}{l}\text { R- } \\
\text { SWT }\end{array}$ & $\begin{array}{l}\text { low-level laser } \\
\text { therapy (LLLT) } \\
\text { and therapeutic } \\
\text { ultrasound (US) }\end{array}$ & $\begin{array}{l}\text { The maximum thickness of the } \\
\text { proximal PF where it attaches to the } \\
\text { calcaneus was measured using } \\
\text { electronic calipers on fluid-sensitive } \\
\text { MRI sequences in the sagittal and cor- } \\
\text { onal planes. The intrafacial and perifa- } \\
\text { cial soft tissue edema and calcaneal } \\
\text { BME were assessed in the sagittal } \\
\text { plane on short tau inversion recovery } \\
\text { sequences, and the presence of the } \\
\text { calcaneal spurs was evaluated on T1- } \\
\text { weighted sequences }\end{array}$ & $\begin{array}{l}1 \\
\text { month }\end{array}$ & $\begin{array}{l}\text { The mean PFT in the SWT group } \\
\text { changed from } 5.17 \pm 0.89 \text { to } \\
4.31 \pm 0.82 \text {, in the LLLT group from } \\
4.33 \pm 0.59 \text { to } 3.75 \pm 0.69 \text {, and in } \\
\text { the US group, from } 4.76 \pm 0.72 \text { to } \\
3.99 \pm 0.62 \text { seen on MRI coronal } \\
\text { plane }\end{array}$ & $<.01$ & NS \\
\hline $\begin{array}{l}\text { Vahdatpour } \\
\text { (2012) [56] }\end{array}$ & $\begin{array}{l}\text { F- } \\
\text { SWT } \\
\text { and } \\
\text { R- } \\
\text { SWT }\end{array}$ & Placebo & $\begin{array}{l}\text { Sagittal imaging of the PF was } \\
\text { performed with the ultrasound } \\
\text { transducer aligned along the } \\
\text { longitudinal axis of the aponeurosis. } \\
\text { PFT was measured about } 2 \mathrm{~cm} \text { distal } \\
\text { of the medial calcaneal tuberosity. } \\
\text { Qualitative assessment was performed } \\
\text { including echogenic appearance of } \\
\text { plantar fascia and its fibrillary pattern }\end{array}$ & $\begin{array}{l}3 \\
\text { months }\end{array}$ & $\begin{array}{l}\text { The mean PFT in the SWT group } \\
\text { changed from } 4.1 \pm 1.3 \text { to } 3.6 \pm 1.2 \text {, } \\
\text { in the placebo group from } 4.1 \pm \\
0.8 \text { to } 4.5 \pm 0.9\end{array}$ & $<.01$ & $=0.02$ \\
\hline $\begin{array}{l}\text { Yalcin (2012) } \\
\text { [57] }\end{array}$ & $\begin{array}{l}\text { R- } \\
\text { SWT }\end{array}$ & None & $\begin{array}{l}\text { Lateral radiographs evaluated } \\
\text { variations in the dimensions of } \\
\text { calcaneal spurs. The radiographic } \\
\text { variations included classification as } \\
\text { reductions in the dimensions and the }\end{array}$ & NR & $\begin{array}{l}\text { No significant disappearance of } \\
\text { heel spurs, but } 19(17.6 \%) \text { had a } \\
\text { decrease in the angle of the spur, } \\
23(21.3 \%) \text { had a decrease in the } \\
\text { dimensions of the spur, and }\end{array}$ & NR & NA \\
\hline
\end{tabular}


Table 4 Imaging outcome measures for plantar fasciitis and heel spurs (Continued)

\begin{tabular}{|c|c|c|c|c|c|c|c|}
\hline \multirow[t]{3}{*}{ Author (year) } & \multirow{3}{*}{$\begin{array}{l}\text { SWT } \\
\text { type }\end{array}$} & \multirow[t]{3}{*}{ Comparator } & \multirow[t]{3}{*}{ Imaging outcome } & \multicolumn{4}{|c|}{ Follow-up } \\
\hline & & & & \multirow[t]{2}{*}{ Period } & \multirow{2}{*}{$\begin{array}{l}\text { Baseline - F/U } \\
\text { Mean } \pm \text { SD }\end{array}$} & \multicolumn{2}{|l|}{$\boldsymbol{P}$ value } \\
\hline & & & & & & $\begin{array}{l}\text { Within } \\
\text { group }\end{array}$ & $\begin{array}{l}\text { Between } \\
\text { group }\end{array}$ \\
\hline & & & angle of calcaneal spurs & & $1(0.93 \%)$ had breakage of the spur & & \\
\hline $\begin{array}{l}\text { Zhu (2005) } \\
\text { [58] }\end{array}$ & $\begin{array}{l}\text { F- } \\
\text { SWT }\end{array}$ & None & $\begin{array}{l}\text { Prior to MRI, a vitamin E capsule was } \\
\text { taped to the heel that could be } \\
\text { readily seen on MRI, where the point } \\
\text { of maximal intensity of pain was } \\
\text { delineated with a permanent marker. } \\
\text { MRI assessed the presence and } \\
\text { severity of soft tissue and calcaneal } \\
\text { marrow edema, heel spur and PFT. }\end{array}$ & $24 \mathrm{~h}$ & $\begin{array}{l}\text { 16/18(89\%) had subcutaneous soft } \\
\text { tissue and perifascial edema } \\
\text { before SWT. After SWT, all } 18 \\
\text { showed subcutaneous soft tissue } \\
\text { and perifascial edema. Calcaneal } \\
\text { marrow edema was seen in } 8 \\
\text { heels. After SWT, edema increased } \\
\text { in } 1 \text { heel and } 1 \text { new heel edema } \\
\text { was developed. } \\
\text { Heel spur was seen in 9(50\%) that } \\
\text { was unchanged. } \\
17(94 \%) \text { had an abnormal PFT (> } \\
4 \text { mm) before SWT that remained } \\
\text { unchanged following SWT }\end{array}$ & NR & NA \\
\hline
\end{tabular}

BME bone marrow edema, F-SWT focused SWT, R-SWT radial SWT, NA not applicable, NR not reported, NS not significant

regression analyses. Also, the utilization of imaging guidance and use of anesthesia had no clear impact on the evaluated imaging outcomes. The baseline size of the lesion was the only factor that explained the heterogeneity in our findings. However, these results should be interpreted with caution due to the relatively small overall number of studies and several potential sources of heterogeneity such as variation in study design, variation in imaging methods and measures, time period for imaging follow-up, high risk of bias and the small number of trials included in the subgroup and metaregression analyses. In addition, SWT device related factors may contribute to the heterogeneity such as different SWT types. Further research is required to clearly determine whether there are differences in response to focused or radial SWT for different conditions.

Our meta-analysis data on the effect of SWT on rotator cuff calcific tendinitis were comparable to a recent meta-analysis [4] that reported total resorption occurred more commonly using high- versus low-energy SWT at 3 months (OR: 3.4 (95\% CI 1.35,8.58); $p=0.009$ ) based on 3 included studies of 163 participants. In addition to reporting the chances of total resorption versus control, the current meta-analysis has also reported within and between groups changes in the size of the calcium deposit diameter that have not been reported previously. A small number of studies $(5 / 23)$ reported quantification of the change in the size

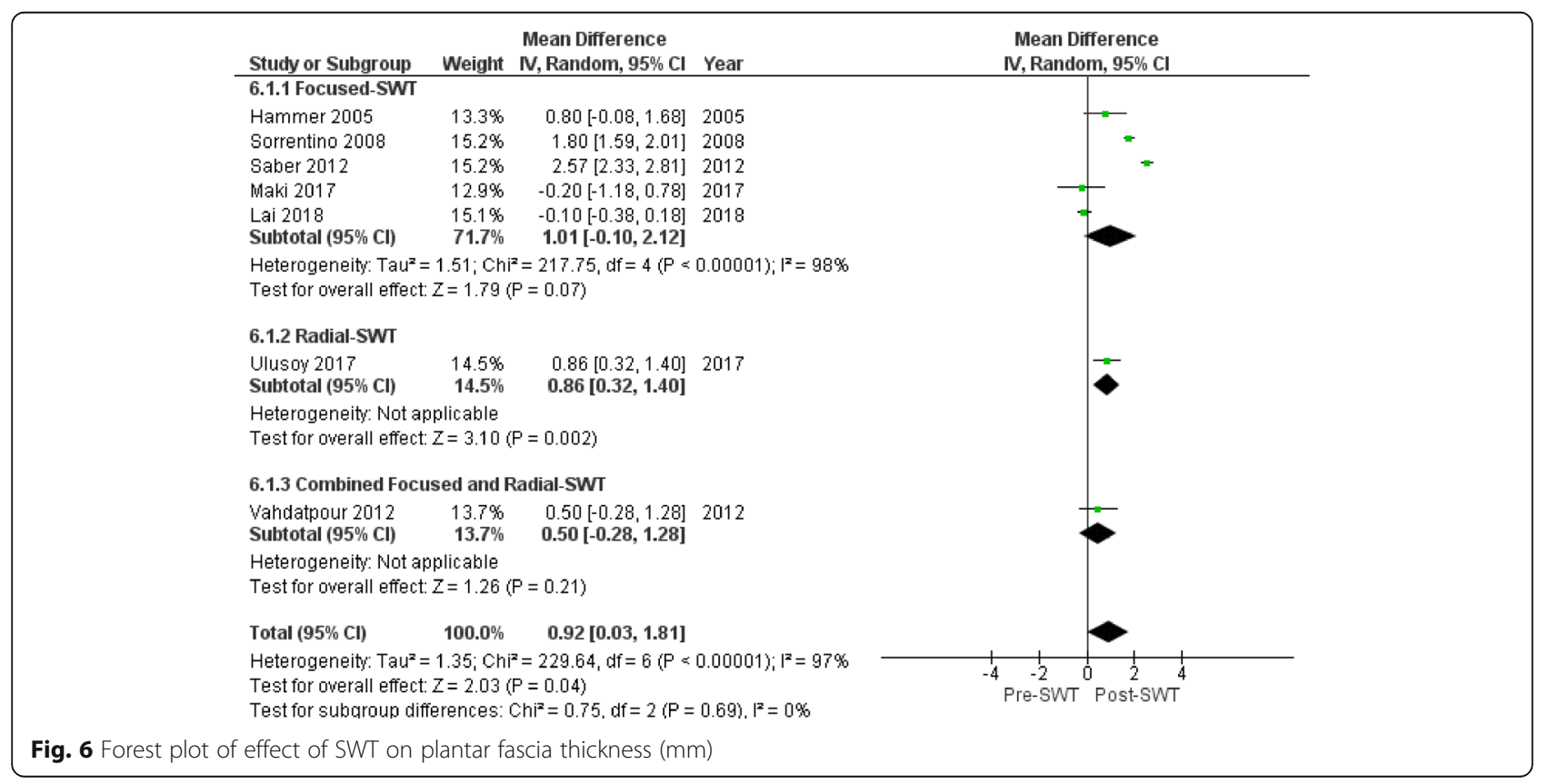




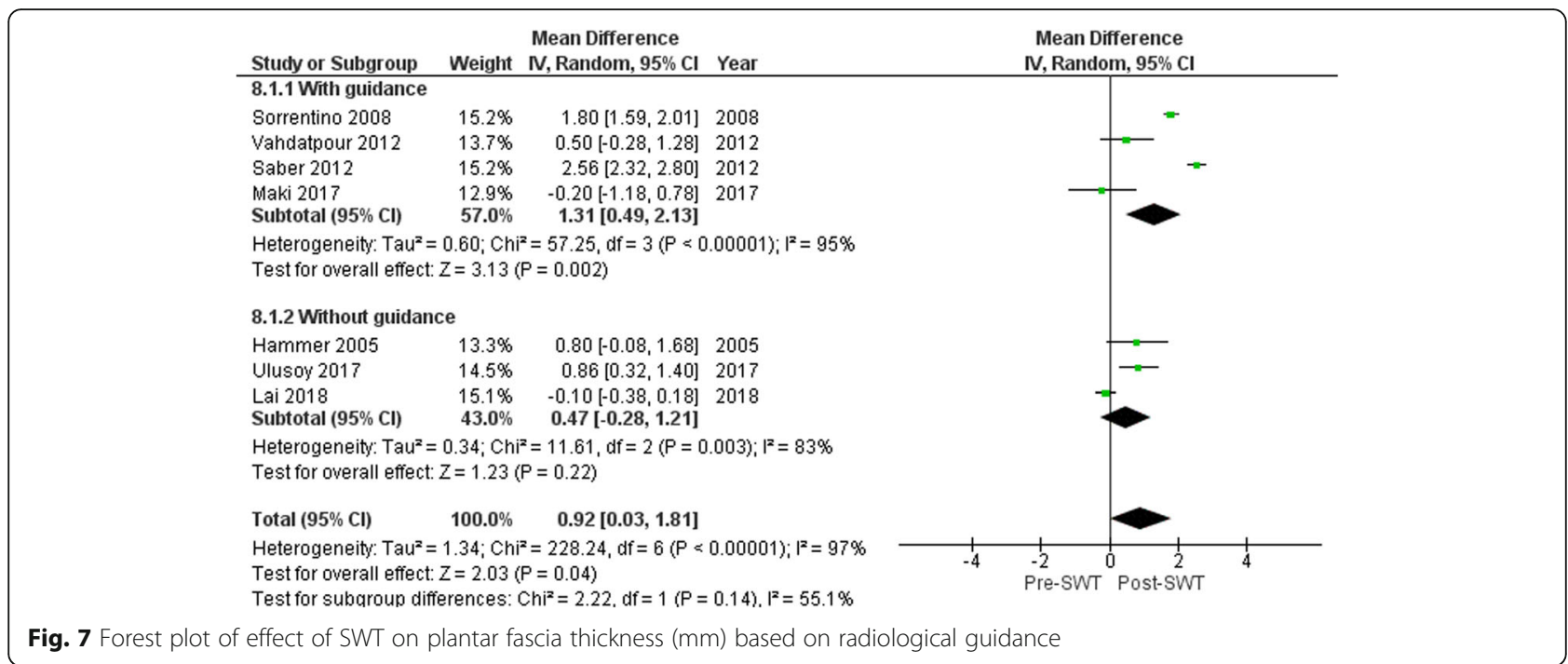

of calcium deposit diameter, which limited the power of the current meta-analysis. Out of the five included studies in the meta-analysis, Cacchio et al. [21] reported the highest rate of total resorption (86.6\%) and reduction in calcification deposit size $\mathrm{MD}=20.45 \mathrm{~mm}(18.23,22.67)$ at one week follow-up using a radial SWT device. The authors themselves did not expect this high rate of resorption and attributed it to the feature of radial SWT that insures the whole calcification area is included inside the wave propagation area. The only available included comparable study is by De Boer et al. [25] that also used a radial SWT device with similar treatment parameters demonstrating a total resorption of only $7 \%$ at six weeks. Our data comparison could provide an explanation related to the initial size of the calcium deposit that was exceptionally high in the Cacchio et al. [21] study $(21.3 \pm 7.5 \mathrm{~mm})$, which was the only significant predictor in our meta-regression analysis. Larger deposits being more responsive to treatment.

According to our meta-analysis data, SWT demonstrated significant reduction in PFT and associated calcaneal BME. The reduction in PFT was greater when utilizing radiological guidance, which might be due to more consistent targeting of the SWT to the affected tissue area and

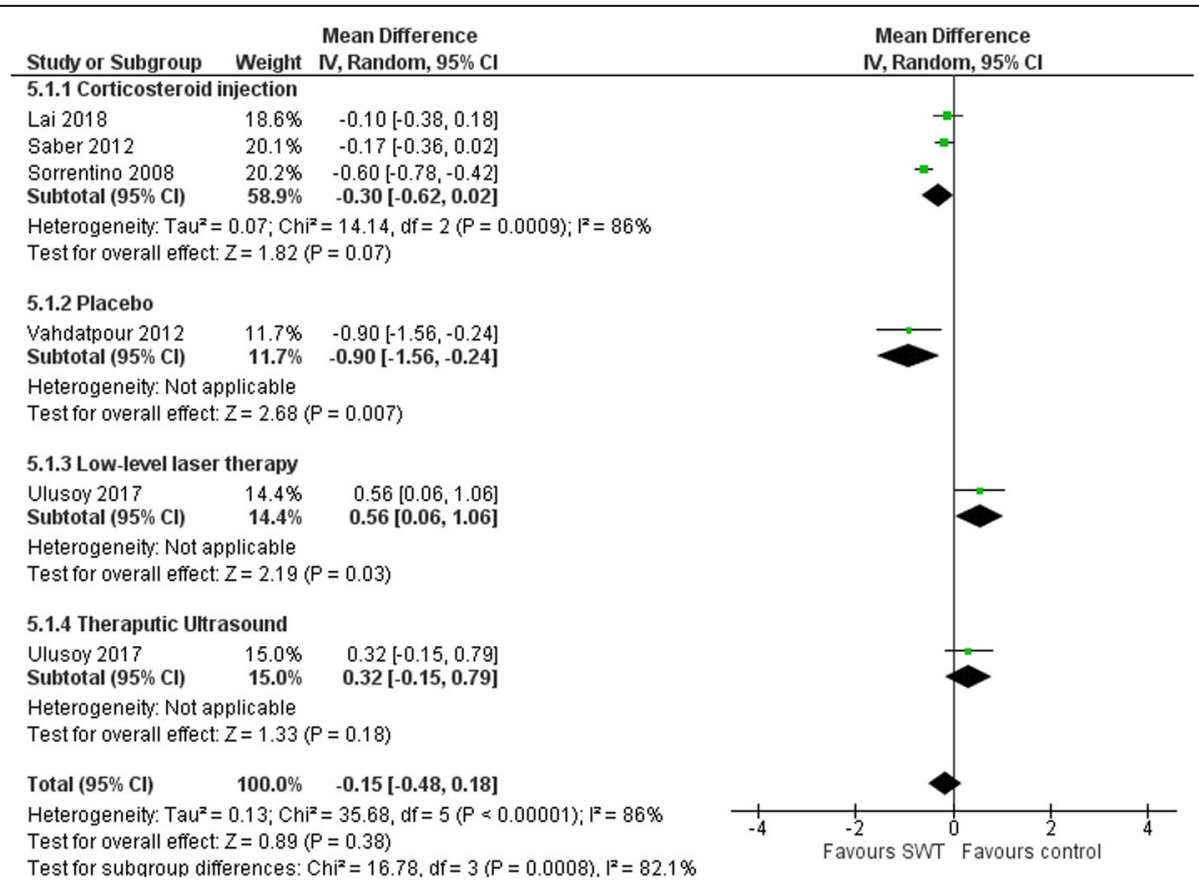

Fig. 8 Forest plot of effect of SWT vs control on plantar fascia thickness ( $\mathrm{mm}$ ) 
Table 5 Characteristics of studies and intervention details for osteonecrosis of the femoral head (ONFH)

\begin{tabular}{|c|c|c|c|c|c|c|c|c|c|}
\hline $\begin{array}{l}\text { Author } \\
\text { (year) }\end{array}$ & Study design & $\mathrm{N}$ & $\begin{array}{l}\text { Mean } \\
\text { age } \\
\pm \text { SD or } \\
\text { (range) }\end{array}$ & $\begin{array}{l}\text { Mean } \\
\text { symptoms } \\
\text { duration } \pm \\
\text { SD or } \\
\text { (range), } \\
\text { months }\end{array}$ & Area of SWT application & $\begin{array}{l}\text { Dosage in } \\
\text { impulses*EFD } \\
\left(\mathrm{mJ} / \mathrm{mm}^{2}\right) / \mathrm{bar}\end{array}$ & $\begin{array}{l}\text { No. of } \\
\text { sessions }\end{array}$ & $\begin{array}{l}\text { Interval } \\
\text { between } \\
\text { sessions }\end{array}$ & $\begin{array}{l}\text { Co- } \\
\text { intervention, } \\
\text { anesthesia }\end{array}$ \\
\hline $\begin{array}{l}\text { Algarni } \\
\text { (2018) [61] }\end{array}$ & $\begin{array}{l}\text { Prospective } \\
\text { open design }\end{array}$ & $\begin{array}{l}21 \text { (33 } \\
\text { hips) }\end{array}$ & $\begin{array}{l}37.5 \pm \\
4.8\end{array}$ & $6 \pm 3$ & $\begin{array}{l}\text { The hip was fixed in adduction } \\
\text { and internal rotation, ONFH was } \\
\text { marked using fluoroscopy in } 2-3 \\
\text { points depending on the size of } \\
\text { the lesion }\end{array}$ & $\begin{array}{l}3000-4500 \\
(1500 \text { pulses } \\
\text { for each } 2-3 \\
\text { point })^{*} 26 \mathrm{kV}\end{array}$ & 1 & NA & None, yes \\
\hline $\begin{array}{l}\text { Chen } \\
\text { (2009) [62] }\end{array}$ & $\begin{array}{l}\text { Prospective } \\
\text { comparative } \\
\text { design }\end{array}$ & 17 & $\begin{array}{l}42.9 \pm \\
9.3\end{array}$ & $11.3 \pm 3.4$ & $\begin{array}{l}\text { Four points with } 1 \mathrm{~cm} \text { apart } \\
\text { within the junctional zone were } \\
\text { chosen with a metallic pin } \\
\text { under C-arm control, and the } \\
\text { corresponding locations were } \\
\text { marked on the skin in the groin } \\
\text { area. The depth of treatment } \\
\text { was adjusted until the two ring } \\
\text { markers of the device synchro- } \\
\text { nized under C-arm imaging }\end{array}$ & $\begin{array}{l}1500^{*} 0.62 \\
\text { each of the } \\
\text { four sites }\end{array}$ & 1 & NA & None, yes \\
\hline $\begin{array}{l}\text { D'Agostino } \\
\text { (2014) [59] }\end{array}$ & $\begin{array}{l}\text { Prospective } \\
\text { open design }\end{array}$ & 20 & 43.23 & $\begin{array}{l}4.2(4-7) \\
\text { weeks }\end{array}$ & NR & $4000 * 0.5$ & 2 & 2 days & None \\
\hline $\begin{array}{l}\text { Hsu (2010) } \\
\text { [63] }\end{array}$ & $\mathrm{RCT}$ & $\begin{array}{l}35 \text { ( } 48 \\
\text { hips) }\end{array}$ & $\begin{array}{l}39.6 \pm \\
11.9\end{array}$ & $7.2 \pm 2.9$ & $\begin{array}{l}\text { The hip joint was properly } \\
\text { positioned by abduction and } \\
\text { internal or external rotation. The } \\
\text { junctional zone between } \\
\text { avascular and normal bones of } \\
\text { the femoral head was } \\
\text { delineated with C-arm imaging }\end{array}$ & $\begin{array}{l}1500^{*} 0.62 \\
\text { each of the } \\
\text { four sites }\end{array}$ & 1 & NA & None, yes \\
\hline $\begin{array}{l}\text { Ludwig } \\
\text { (2001) [64] }\end{array}$ & $\begin{array}{l}\text { Prospective } \\
\text { open design }\end{array}$ & 22 & $\begin{array}{l}54.9 \pm \\
12.3\end{array}$ & NR & NR & $4000 * 0.62$ & 1 & NA & None \\
\hline $\begin{array}{l}\text { Vulpiani } \\
\text { (2012) [60] }\end{array}$ & $\begin{array}{l}\text { Prospective } \\
\text { open design }\end{array}$ & 36 & $\begin{array}{l}\text { Stage I: } \\
49.3 \pm \\
11.9 \\
\text { Stage } \\
\text { II: } \\
52.7 \pm \\
14.6 \\
\text { Stage } \\
\text { III: } \\
45.9 \pm \\
14.1\end{array}$ & $\begin{array}{l}\text { Stage I: } \\
4.3 \pm 2.4 \\
\text { Stage II: } \\
9.3 \pm 4.6 \\
\text { Stage III: } \\
14.7 \pm 5.9\end{array}$ & $\begin{array}{l}\text { SWT was focused around (on } \\
\text { the margins of) the necrotic } \\
\text { bone of the femoral head under } \\
\text { radiographic guidance }\end{array}$ & $2400 * 5$ & 4 & 2-3 days & None \\
\hline $\begin{array}{l}\text { Wang } \\
\text { (2016) [65] }\end{array}$ & RCT & $\begin{array}{l}33(42 \\
\text { hips) }\end{array}$ & $\begin{array}{l}41.8 \pm \\
9.1\end{array}$ & $9.3 \pm 8.4$ & $\begin{array}{l}\text { Both legs were properly } \\
\text { positioned. Under C-arm and } \\
\text { MRI guidance, the junctional } \\
\text { zone between normal bone and } \\
\text { necrotic bone within the fem- } \\
\text { oral head was delineated. Within } \\
\text { the junctional zone, four points } \\
\text { approximately } 1 \mathrm{~cm} \text { apart were } \\
\text { chosen under C-arm imaging } \\
\text { control and the corresponding } \\
\text { locations were marked on the } \\
\text { skin in the groin area }\end{array}$ & $\begin{array}{l}\text { Group A: } \\
2000^{*} 0.51 \\
\text { Group B: } \\
4000^{*} 0.51 \\
\text { Group C: } \\
6000^{*} 0.51\end{array}$ & 1 & NA & None, Yes \\
\hline $\begin{array}{l}\text { Wang } \\
(2012) \text { [66] }\end{array}$ & $\begin{array}{l}\text { Prospective } \\
\text { comparative } \\
\text { design }\end{array}$ & $\begin{array}{l}23(29 \\
\text { hips) }\end{array}$ & NR & NR & NR & $6000 * 0.474$ & 1 & NA & None \\
\hline $\begin{array}{l}\text { Wang } \\
\text { (2009) [67] }\end{array}$ & $\begin{array}{l}\text { Prospective } \\
\text { comparative } \\
\text { design }\end{array}$ & $\begin{array}{l}\text { Total } 39, \\
15(26 \text { hips }) \\
\text { with SLE, } \\
24(29 \text { hips }) \\
\text { controls }\end{array}$ & $\begin{array}{l}\text { SLE } \\
\text { group: } \\
32.33 \pm \\
8.97 \\
\text { Non- } \\
\text { SLE } \\
\text { group: }\end{array}$ & $\begin{array}{l}\text { SLE group: } \\
6.88 \pm 2.63 \\
\text { Non-SLE } \\
\text { group: } \\
7.1 \pm 2.79\end{array}$ & $\begin{array}{l}\text { Four points with } 1 \mathrm{~cm} \text { apart } \\
\text { within the junctional zone were } \\
\text { chosen with a metallic pin } \\
\text { under C-arm control, and the } \\
\text { corresponding locations were } \\
\text { marked on the skin in the groin } \\
\text { area. The depth of treatment }\end{array}$ & $\begin{array}{l}1500^{*} 0.62 \\
\text { each of the } \\
\text { four sites }\end{array}$ & 1 & NA & None, yes \\
\hline
\end{tabular}


Table 5 Characteristics of studies and intervention details for osteonecrosis of the femoral head (ONFH) (Continued)

\begin{tabular}{|c|c|c|c|c|c|c|c|c|c|}
\hline $\begin{array}{l}\text { Author } \\
\text { (year) }\end{array}$ & Study design & $\mathrm{N}$ & $\begin{array}{l}\text { Mean } \\
\text { age } \\
\pm \text { SD or } \\
\text { (range) }\end{array}$ & $\begin{array}{l}\text { Mean } \\
\text { symptoms } \\
\text { duration } \pm \\
\text { SD or } \\
\text { (range), } \\
\text { months }\end{array}$ & Area of SWT application & $\begin{array}{l}\text { Dosage in } \\
\mathrm{impulses}^{*} \mathrm{EFD} \\
\left(\mathrm{mJ} / \mathrm{mm}^{2}\right) / \mathrm{bar}\end{array}$ & $\begin{array}{l}\text { No. of } \\
\text { sessions }\end{array}$ & $\begin{array}{l}\text { Interval } \\
\text { between } \\
\text { sessions }\end{array}$ & $\begin{array}{l}\text { Co- } \\
\text { intervention, } \\
\text { anesthesia }\end{array}$ \\
\hline & & & $\begin{array}{l}36.47 \pm \\
8.95\end{array}$ & & $\begin{array}{l}\text { was adjusted until the two ring } \\
\text { markers of the device synchro- } \\
\text { nized under C-arm imaging }\end{array}$ & & & & \\
\hline $\begin{array}{l}\text { Wang } \\
\text { (2005) [68] }\end{array}$ & $\mathrm{RCT}$ & 23(29 hips) & $\begin{array}{l}39.8 \pm \\
12.1\end{array}$ & $5.9 \pm 4.5$ & $\begin{array}{l}\text { SWT was applied in the supine } \\
\text { position. The hip was positioned } \\
\text { in adduction and internal } \\
\text { rotation. In patients with a } \\
\text { stage-II or III lesion, the junc- } \\
\text { tional zone between avascular } \\
\text { and vascular bone of the fem- } \\
\text { oral head was delineated under } \\
c \text {-arm control. Four focal points } \\
1 \mathrm{~cm} \text { apart, within the junctional } \\
\text { zone were selected, and the cor- } \\
\text { responding locations on the skin } \\
\text { in the groin area were marked. } \\
\text { In patients with a stage-l lesion, } \\
\text { the junctional zone was selected } \\
\text { on the basis of findings on MRI }\end{array}$ & $\begin{array}{l}1500^{*} 0.62 \\
\text { each of the } \\
\text { four sites }\end{array}$ & 1 & NA & None, Yes \\
\hline $\begin{array}{l}\text { Wang } \\
\text { (2008) [69] }\end{array}$ & RCT & $\begin{array}{l}25 \text { ( } 30 \\
\text { hips) }\end{array}$ & $\begin{array}{l}38.6 \pm \\
12.6\end{array}$ & $7.5 \pm 3$ & $\begin{array}{l}\text { The junctional zone between } \\
\text { the avascular and normal bones } \\
\text { of the femoral head was } \\
\text { delineated with C-arm imaging. } \\
\text { Four points with } 1 \mathrm{~cm} \text { apart } \\
\text { within the junctional zone were } \\
\text { chosen with a metallic pin } \\
\text { under C-arm control, and the } \\
\text { corresponding locations were } \\
\text { marked on skin in the groin }\end{array}$ & $\begin{array}{l}1500 * 0.62 \\
\text { each of the } \\
\text { four sites }\end{array}$ & 1 & NA & None, yes \\
\hline $\begin{array}{l}\text { Wang } \\
(2011) \text { [70] }\end{array}$ & $\begin{array}{l}\text { Prospective } \\
\text { open design }\end{array}$ & $\begin{array}{l}35(47 \\
\text { hips) }\end{array}$ & $\begin{array}{l}38.8 \pm \\
11.9\end{array}$ & $7.4 \pm 3$ & $\begin{array}{l}\text { The hip joint was properly } \\
\text { positioned by adduction and } \\
\text { internal or external rotation. } \\
\text { Four points with } 1 \mathrm{~cm} \text { apart } \\
\text { within the junctional zone were } \\
\text { chosen with a metallic pin } \\
\text { under C-arm control, and the } \\
\text { corresponding locations were } \\
\text { marked on the skin in the groin } \\
\text { area. The depth of treatment } \\
\text { was adjusted until the two ring } \\
\text { markers of the device synchro- } \\
\text { nized under C-arm imaging }\end{array}$ & $\begin{array}{l}1500^{*} 0.62 \\
\text { each of the } \\
\text { four sites }\end{array}$ & 1 & NA & None, yes \\
\hline
\end{tabular}

avoiding surrounding areas. Although one study [55] using radial SWT showed greater reduction in PFT over focused or combined SWT, these results should be interpreted with caution based on the small number of studies included in the subgroup analysis. Despite the observed overall reduction in PFT in our review that can be correlated with the improvement in chronic plantar pain [9], it remains unclear whether the type of SWT generation device is an important factor for providing the best outcomes [2].

Our meta-analysis data revealed non-significant reduction in the lesion size with high-over medium and lowdosage SWT for ONFH. The SWT dose parameters were fairly consistent among all the included studies.
This could be attributed to the same research group implementing similar treatment protocols. There are no previously published reviews or meta-analyses reporting on imaging changes following SWT among patients with ONFH to allow results comparisons. However, the observed modest reduction in the size of the lesion could be correlated with a recently published meta-analysis [8] that reported significant differences between SWT and control groups in pain rating and motor function measures.

Overall, the reported studies support changes in morphological features based on imaging findings that may reflect changes in underlying 
Table 6 Imaging outcome measures for osteonecrosis of the femoral head (ONFH)

\begin{tabular}{|c|c|c|c|c|c|c|c|}
\hline \multirow{3}{*}{$\begin{array}{l}\text { Author } \\
\text { (year) }\end{array}$} & \multirow{3}{*}{$\begin{array}{l}\text { SWT } \\
\text { type }\end{array}$} & \multirow[t]{3}{*}{ Comparator } & \multirow[t]{3}{*}{ Imaging outcome } & \multicolumn{4}{|c|}{ Follow-up } \\
\hline & & & & \multirow[t]{2}{*}{ Period } & \multirow{2}{*}{$\begin{array}{l}\text { Baseline - F/U } \\
\text { Mean } \pm \text { SD }\end{array}$} & \multicolumn{2}{|l|}{$\boldsymbol{P}$ value } \\
\hline & & & & & & Within group & $\begin{array}{l}\text { Between } \\
\text { group }\end{array}$ \\
\hline $\begin{array}{l}\text { Algarni } \\
(2018)[61]\end{array}$ & $\begin{array}{l}\text { F- } \\
\text { SWT }\end{array}$ & None & $\begin{array}{l}\text { Anteroposterior and lateral } \\
\text { radiographs were obtained to assess } \\
\text { the size of the lesion, the extent of } \\
\text { subchondral bone collapse, and the } \\
\text { presence of degenerative changes in } \\
\text { the hip joint. MRI was performed to } \\
\text { evaluate BME, the size of the lesion, } \\
\text { femoral head congruency, the } \\
\text { presence of a crescent sign, and } \\
\text { degenerative changes in the hip joint }\end{array}$ & $\begin{array}{l}6,12,24 \\
\text { months }\end{array}$ & $\begin{array}{l}\text { The mean size of the lesion (\%) over } \\
\text { the femoral head pre-SWT was } 59 \pm \\
32 \text { and post-SWT was } 28 \pm 16 \text {. } \\
\text { Significant reduction in BME was } \\
\text { noted following SWT }\end{array}$ & $p=0.24$ & NA \\
\hline $\begin{array}{l}\text { Chen } \\
\text { (2009) [62] }\end{array}$ & $\begin{array}{l}\text { F- } \\
\text { SWT }\end{array}$ & $\begin{array}{l}\text { Total hip } \\
\text { arthroplasty }\end{array}$ & $\begin{array}{l}\text { MRI were assessed for the } \\
\text { congruency of the femoral head, } \\
\text { crescent sign, the size and stage of } \\
\text { the lesion and bone marrow edema }\end{array}$ & $\begin{array}{l}41 \pm \\
7.4 \\
\text { month }\end{array}$ & $\begin{array}{l}\text { The mean size of the lesion (\%) over } \\
\text { the femoral head pre-SWT was } \\
23.1 \pm 22.2 \text { and post-SWT was } 22 \pm \\
23.3 \text {. } \\
\text { Significant reduction in BME was } \\
\text { noted after treatment }\end{array}$ & $\begin{array}{l}\text { lesion size }= \\
0.466 \\
\text { BME }=0.031\end{array}$ & NA \\
\hline $\begin{array}{l}\text { D'Agostino } \\
\text { (2014) [59] }\end{array}$ & $\begin{array}{l}\text { F- } \\
\text { SWT }\end{array}$ & None & $\begin{array}{l}\text { MRI examination was performed and } \\
\text { calculated the edema area using the } \\
\text { Sectra PACS software }\end{array}$ & $\begin{array}{l}2,3,6 \\
\text { months }\end{array}$ & $\begin{array}{l}\text { Pre-treatment, the mean edema area } \\
(\mathrm{mm} 2) \text { was } 981.9 \pm 453.2 \text {. After } 2 \\
\text { months was } 469.5 \pm 306.8 \text {. At } 6 \\
\text { months, the mean edema area had } \\
\text { reduced to } 107.8 \pm 248.1\end{array}$ & $<0.01$ & NA \\
\hline $\begin{array}{l}\text { Hsu (2010) } \\
{[63]}\end{array}$ & $\begin{array}{l}\text { F- } \\
\text { SWT }\end{array}$ & $\begin{array}{l}\text { Cocktail therapy } \\
\text { (SWT, hyperbaric } \\
\text { oxygen therapy } \\
\text { (HBO), } \\
\text { alendronate) }\end{array}$ & $\begin{array}{l}\text { Radiographs were used to assess the } \\
\text { size and location of the lesion, } \\
\text { congruency of the femoral head, the } \\
\text { presence of a crescent sign and } \\
\text { degenerative changes of the hip joint. } \\
\text { MRI was used to evaluate the } \\
\text { changes in the size of the lesion, the } \\
\text { congruency of the articular surface of } \\
\text { the femoral head and BME }\end{array}$ & $\begin{array}{l}6,12 \\
\text { months }\end{array}$ & $\begin{array}{l}\text { The mean size of the lesion (\%) over } \\
\text { the total femoral head surface was } \\
28.9 \pm 14.9 \text { and } 27.4 \pm 18 \text { before } \\
\text { treatment, and } 27.6 \pm 14.5 \text { and } \\
26.2 \pm 18.5 \text { after treatment for the } \\
\text { Cocktail therapy group and SWT } \\
\text { alone group, respectively }\end{array}$ & $\begin{array}{l}=0.373 \text { for } \\
\text { the lesion } \\
\text { size, }=0.033 \\
\text { for the BME }\end{array}$ & $=0.344$ \\
\hline $\begin{array}{l}\text { Ludwig } \\
\text { (2001) [64] }\end{array}$ & $\begin{array}{l}\text { F- } \\
\text { SWT }\end{array}$ & None & $\begin{array}{l}\text { Radiography and MRI were used to } \\
\text { classify the lesions on the ARCO scale }\end{array}$ & 1 year & $\begin{array}{l}\text { complete healing in } 4 \text { patients, a } \\
\text { significant decrease in the size of } \\
\text { the area of poor circulation in } 6 \\
\text { patients, and unchanged in } 4 \\
\text { patients }\end{array}$ & NR & NA \\
\hline $\begin{array}{l}\text { Vulpiani } \\
\text { (2012) [60] }\end{array}$ & $\begin{array}{l}\text { F- } \\
\text { SWT }\end{array}$ & None & $\begin{array}{l}\text { Antero-posterior and lateral } \\
\text { radiographs were used to evaluate } \\
\text { the size of the lesion, the extent of } \\
\text { collapse of subchondral bone and } \\
\text { degenerative changes of the hip joint. } \\
\text { MRI was used to measure the size of } \\
\text { the lesion, assess the congruency of } \\
\text { the femoral head, the presence of a } \\
\text { crescent sign and/or degenerative } \\
\text { changes, with the aim to stage the } \\
\text { lesion according to ARCO scale }\end{array}$ & $\begin{array}{l}3,6,12, \\
24 \\
\text { months }\end{array}$ & $\begin{array}{l}\text { At all follow-up time points, the le- } \\
\text { sions show no or only minimal } \\
\text { changes. Neither regression nor pro- } \\
\text { gression of lesions that had been } \\
\text { graded before treatment as ARCO } \\
\text { stage I and II were seen. }\end{array}$ & NR & NA \\
\hline $\begin{array}{l}\text { Wang } \\
(2016)[65]\end{array}$ & $\begin{array}{l}\text { F- } \\
\text { SWT }\end{array}$ & $\begin{array}{l}\text { Low vs medium } \\
\text { vs high dosage } \\
\text { SWT }\end{array}$ & $\begin{array}{l}\text { The necrotic areas of femoral heads } \\
\text { on MRI were estimated on a high } \\
\text { resolution monitor via the PACS } \\
\text { system. The percentage of the } \\
\text { infarcted femoral head volume (IFHV) } \\
\text { was measured by the infarcted } \\
\text { femoral head volume divided by total } \\
\text { femoral head volume. BME around } \\
\text { the necrotic regions were graded on } \\
\text { MRI as follows: grade } 0 \text { : no BME; } \\
\text { grade } 1 \text { : peri-necrotic; grade } 2 \text { : edema } \\
\text { extending to femoral head; grade } 3 \text { : } \\
\text { edema extending to femoral neck } \\
\text { and grade } 4 \text { : edema extending to } \\
\text { intertrochanteric region }\end{array}$ & $\begin{array}{l}6 \\
\text { months }\end{array}$ & $\begin{array}{l}\text { The mean size of the lesion (\%) in } \\
\text { group A pre-SWT was } 35.1 \pm 9.4 \text { and } \\
\text { post-SWT was } 34.2 \pm 5.9 \text {, group B } \\
\text { pre-SWT was } 36.2 \pm 8.6 \text { and post- } \\
\text { SWT was } 36.6 \pm 7.7 \text { and group C pre- } \\
\text { SWT was } 30.5 \pm 13.1 \text { and post-SWT } \\
\text { was } 30.2 \pm 7.3 \text {. } \\
\text { The IFHV of lesion (\%) in group A at } \\
\text { pre-SWT was } 20.8 \pm 18.7 \text { and post- } \\
\text { SWT was } 19.3 \pm 19 \text {, group B at pre- } \\
\text { SWT was } 23 \pm 14.1 \text { and post-SWT } \\
\text { was } 22.5 \pm 16.4 \text {, and group C at pre- } \\
\text { SWT was } 22.3 \pm 15.7 \text { and post-SWT } \\
\text { was } 18.9 \pm 12.5 \text {. } \\
\text { The stage of the lesion showed no } \\
\text { significant differences in all groups. } \\
\text { However, BME on MRI was } \\
\text { significantly reduced after SWT in } \\
\text { group C (P=0.039). }\end{array}$ & $\begin{array}{l}>0.05 \text { except } \\
\text { for the IFHV } \\
\text { of lesion in } \\
\text { group C = } \\
0.028\end{array}$ & $>0.05$ \\
\hline
\end{tabular}


Table 6 Imaging outcome measures for osteonecrosis of the femoral head (ONFH) (Continued)

\begin{tabular}{|c|c|c|c|c|c|c|c|}
\hline \multirow{3}{*}{$\begin{array}{l}\text { Author } \\
\text { (year) }\end{array}$} & \multirow{3}{*}{$\begin{array}{l}\text { SWT } \\
\text { type }\end{array}$} & \multirow[t]{3}{*}{ Comparator } & \multirow[t]{3}{*}{ Imaging outcome } & \multicolumn{4}{|c|}{ Follow-up } \\
\hline & & & & \multirow[t]{2}{*}{ Period } & \multirow{2}{*}{$\begin{array}{l}\text { Baseline - F/U } \\
\text { Mean } \pm S D\end{array}$} & \multicolumn{2}{|l|}{$\boldsymbol{P}$ value } \\
\hline & & & & & & Within group & $\begin{array}{l}\text { Between } \\
\text { group }\end{array}$ \\
\hline $\begin{array}{l}\text { Wang } \\
\text { (2012) [66] }\end{array}$ & $\begin{array}{l}\text { F- } \\
\text { SWT }\end{array}$ & $\begin{array}{l}\text { Core } \\
\text { decompression }\end{array}$ & $\begin{array}{l}\text { MRI was used to examine the size of } \\
\text { the lesion, congruency of the femoral } \\
\text { head, the presence of a crescent sign, } \\
\text { BME and degenerative changes of the } \\
\text { hip joint. The percentage of IFHV was } \\
\text { measured by IFHV divided by total } \\
\text { femoral head volume. }\end{array}$ & $\begin{array}{l}1,2,9-8 \\
\text { years }\end{array}$ & $\begin{array}{l}\text { The mean size of the lesion (\%) over } \\
\text { the femoral head pre-SWT was } 21 \pm \\
41 \text { and post-SWT was } 30 \pm 20,30 \pm \\
20,26 \pm 18 \text { at } 1,2,8-9 \text { years respect- } \\
\text { ively. In the pre-surgical group was } \\
40 \pm 23 \text {, and post-surgical was } 42 \pm \\
15,41 \pm 27,41 \pm 4 \text { at } 1,2,8-9 \text { years } \\
\text { respectively. }\end{array}$ & $>0.05$ & $\begin{array}{l}<0.05 \text { for the } \\
\text { size of the } \\
\text { lesion and } \\
\text { reduction of } \\
\text { BME after SWT }\end{array}$ \\
\hline $\begin{array}{l}\text { Wang } \\
\text { (2009) [67] }\end{array}$ & $\begin{array}{l}\text { F- } \\
\text { SWT }\end{array}$ & None & $\begin{array}{l}\text { Radiographs were used to assess the } \\
\text { size of the lesion, congruency of the } \\
\text { femoral head, the presence of a } \\
\text { crescent sign and degenerative } \\
\text { changes. MRI was used to evaluate } \\
\text { the changes in lesion size, the } \\
\text { congruency of the articular surface } \\
\text { and BME. }\end{array}$ & $\begin{array}{l}1,3,6,12 \\
24 \\
\text { months }\end{array}$ & $\begin{array}{l}\text { The mean size of the lesion (\%) over } \\
\text { the femoral head pre-SWT in the SLE } \\
\text { group was } 34.8 \pm 21.1 \text { and } 28.7 \pm \\
14.2 \text { post-SWT, in the Non-SLE } \\
\text { group, pre-SWT was } 32.9 \pm 22.4 \text { and } \\
\text { post-SWT was } 26.7 \pm 12.9 \text {. both } \\
\text { groups showed significant reduction } \\
\text { of BME following SWT }\end{array}$ & $>0.05$ & $\begin{array}{l}>0.05 \text { for the } \\
\text { size of lesion } \\
\text { and }<0.05 \text { for } \\
\text { reduction of } \\
\text { BME }\end{array}$ \\
\hline $\begin{array}{l}\text { Wang } \\
\text { (2005) [68] }\end{array}$ & $\begin{array}{l}\text { F- } \\
\text { SWT }\end{array}$ & $\begin{array}{l}\text { core } \\
\text { decompression } \\
\text { and bone- } \\
\text { grafting }\end{array}$ & $\begin{array}{l}\text { Radiographs of the hip joint were } \\
\text { used to evaluate the size of the } \\
\text { lesion, the extent of collapse of } \\
\text { subchondral bone, and degenerative } \\
\text { changes of the hip joint. MRI was } \\
\text { used to examine the size of the } \\
\text { lesion, the congruency of the femoral } \\
\text { head, the presence of a crescent sign, } \\
\text { BME, and degenerative changes of } \\
\text { the hip joint }\end{array}$ & $\begin{array}{l}3,6,12, \\
24 \\
\text { months }\end{array}$ & $\begin{array}{l}\text { The mean size of the lesion (\%) over } \\
\text { the femoral head pre-SWT was } 61 \pm \\
41 \text { and post-SWT was } 30 \pm 20 \text { at } 24 \\
\text { months follow-up. In contrast, the } \\
\text { pre-surgical was } 40 \pm 23 \text { and post- } \\
\text { surgical was } 41 \pm 27 \text {. } \\
\text { In the SWT group, } 5 \text { lesions ( } 3 \text { stage } \\
\text { I and } 2 \text { stage II) regressed and } 4 \text { ( } 2 \\
\text { stage II and } 2 \text { stage III) progressed. } \\
\text { In the surgical group, } 4 \text { lesions } \\
\text { regressed, } 15 \text { ( } 14 \text { stage II and } 1 \\
\text { stage I) progressed, and } 9 \text { were } \\
\text { unchanged }\end{array}$ & $=0.282$ & $<0.01$ \\
\hline $\begin{array}{l}\text { Wang } \\
\text { (2008) [69] }\end{array}$ & $\begin{array}{l}\text { F- } \\
\text { SWT }\end{array}$ & $\begin{array}{l}\text { SWT + } \\
\text { alendronate }\end{array}$ & $\begin{array}{l}\text { Radiographs of the hip joint were } \\
\text { used to assess the size of the lesion, } \\
\text { congruency of the femoral head, the } \\
\text { presence of a crescent sign and } \\
\text { degenerative changes of the hip joint. } \\
\text { MRI was used to examine the size of } \\
\text { the lesion, the congruency of the } \\
\text { femoral head, the presence of a } \\
\text { crescent sign, BME, and degenerative } \\
\text { changes of the hip joint }\end{array}$ & $\begin{array}{l}3,6,12, \\
24 \\
\text { months }\end{array}$ & $\begin{array}{l}\text { The mean size of the lesion (\%) over } \\
\text { the femoral head pre-SWT was } \\
27.7 \pm 15.5 \text { and post-SWT was } 25.7 \pm \\
16.2 \text { at } 6 \text { months follow-up. In con- } \\
\text { trast, the pre-SWT+ alendronate } \\
\text { group was } 32.6 \pm 19.9 \text { and post- } \\
\text { SWT+ alendronate was } 29.32 \pm 21.99 \text {. } \\
\text { Significant reduction in BME was } \\
\text { noted in both groups. }\end{array}$ & $=0.679$ & 0.145 \\
\hline $\begin{array}{l}\text { Wang } \\
\text { (2011) [70] }\end{array}$ & $\begin{array}{l}\text { F- } \\
\text { SWT }\end{array}$ & None & $\begin{array}{l}\text { Radiographs in AP and lateral views } \\
\text { were used to assess the size of the } \\
\text { lesion, congruency of the femoral } \\
\text { head, the presence of a crescent sign } \\
\text { and degenerative changes. MRI was } \\
\text { used to evaluate the size of the } \\
\text { lesion, the collapse of femoral head } \\
\text { and BME. }\end{array}$ & $\begin{array}{l}6,12 \\
\text { months }\end{array}$ & $\begin{array}{l}\text { The mean size of the lesion (\%) over } \\
\text { the femoral head pre-SWT was } \\
27.23 \pm 18.9 \text { and } 27.04 \pm 19.17 \text { post- } \\
\text { SWT. Significant improvement in } \\
\text { BME was noted following SWT }\end{array}$ & $\begin{array}{l}>0.05 \text { for the } \\
\text { size of lesion } \\
\text { and }=0.04 \text { for } \\
\text { reduction of } \\
\text { BME }\end{array}$ & NA \\
\hline
\end{tabular}

BME bone marrow edema, F-SWT focused SWT, $R$-SWT radial SWT, NR not reported, NS not significant, NA not applicable

pathophysiological processes. It would appear that SWT has a clear influence on the morphology of the reported conditions, although for some conditions there is evidence to suggest that other treatments may have a greater influence on the underlying pathophysiology and associated morphological changes.

\section{Limitations of the study}

Our aim was to include studies on all kinds of relevant musculoskeletal conditions that had been treated with any type of SWT and reporting any imaging outcomes. We conducted a comprehensive search strategy by including all possible synonyms to avoid missing any potential relevant trials, hence reducing publication bias. To date, we are not aware of any previous systematic reviews that evaluated changes in musculoskeletal conditions based on imaging outcomes following SWT interventions. Publication bias was not evaluated as there were $<10$ trials for each included condition; hence, the power of the test would be very low to distinguish real asymmetry from chance.

The risk of bias scores were $60 \%$ for the RCT studies and $74 \%$ for non-RCT studies indicating relatively high risk of bias. Participant blinding, allocation concealment, 


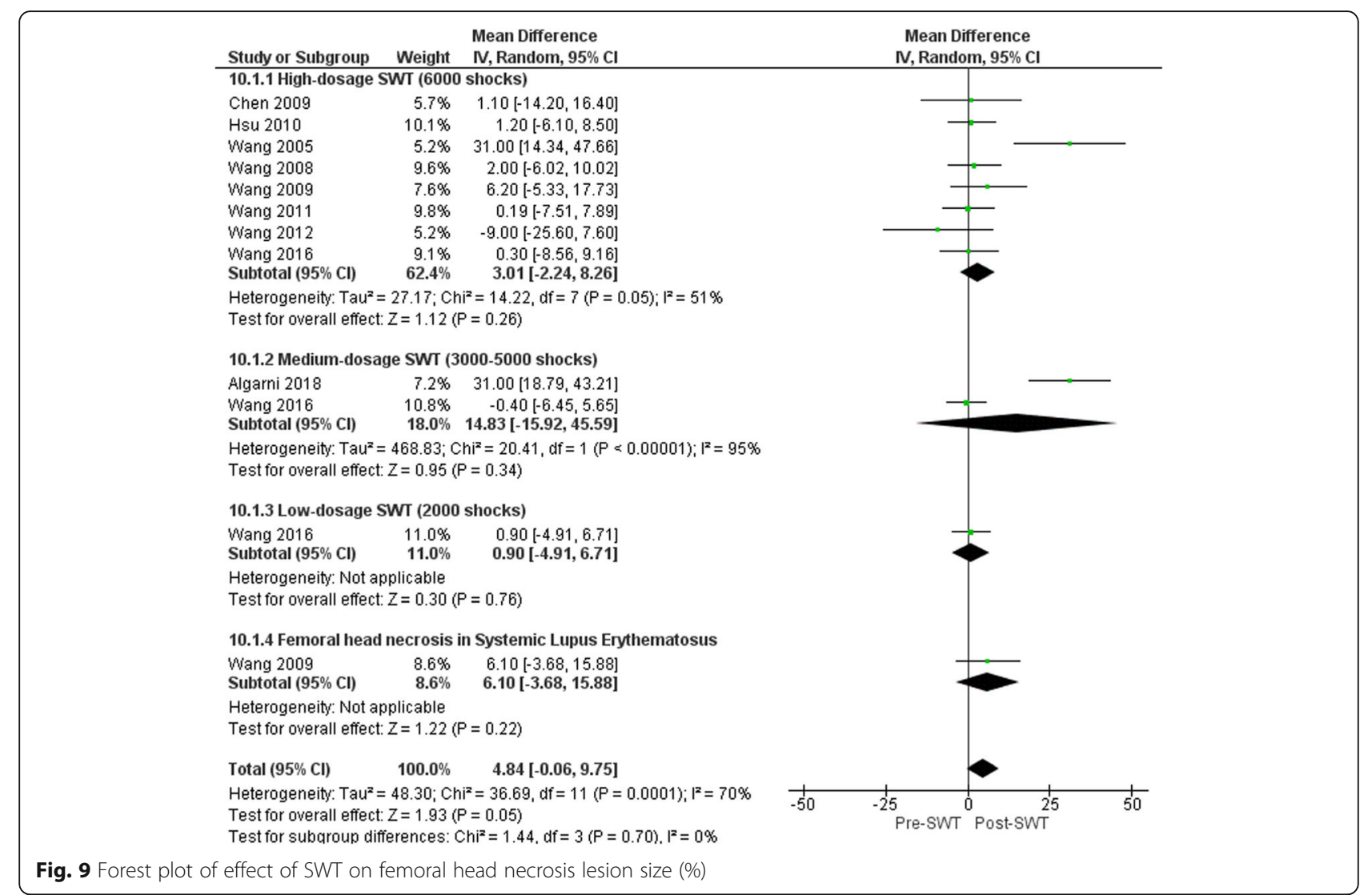

study size calculation and other sources of bias (defined according to imaging assessment accuracy) were the lowest scored items. Of particular interest to this review, the accuracy of imaging measurements was questionable in some cases due to the under-reporting of details pertaining to measurement standardization. It was decided that meeting a minimum of two out of an a priori set of four criteria related to imaging accuracy would be used for judging risk of bias based on imaging accuracy. These criteria were based on providing details on the experience or specialty of the radiologist in musculoskeletal imaging, details of the imaging procedure to insure participant's consistent position during all image acquisition, prior testing or training of the assessor to ensure reliability and reporting the score of measurements based on the average of multiple measurements.

\section{Future research recommendations}

Current available research has provided preliminary evidence related to the capacity of SWT to influence underlying pathophysiological processes in various musculoskeletal conditions as demonstrated through changes in imaging. However, considering more standardized and reliable quantitative imaging measures as a primary outcome would be warranted in future research. This can be achieved through improving the imaging outcomes assessment methodology to ensure consistent and valid reporting based on our suggested criteria for imaging assessment accuracy. Adopting such criteria can limit the imaging assessment procedure variations as it is challenging to account for it as a covariate in the intervention effect estimate. Imaging endpoints are recommended to be specified and reported to evaluate short, medium and long term changes. Study sample sizes should be calculated based upon imaging parameters as a primary outcome. It would also be of great value if researchers could reach consensus on the optimal imaging modality and relevant imaging measures for each musculoskeletal disorder. Consistency of approach would significantly improve the quality of research.

It was surprising that our comprehensive search strategy did not identify any studies using imaging outcomes for commonly treated tendinopathies such as patellar, proximal hamstring and rotator cuff noncalcific tendinopathies and identified only a few trials for Achilles and wrist extensor tendinopathies. This is an indication of the limited use of imaging as a measure of outcome in addition to the usual clinical outcome measures for this type of condition. Whilst clinical outcomes are clearly of primary importance, imaging does provide a window to assist us in understanding the effects and potential mechanism of action of SWT. Future trials might consider making 


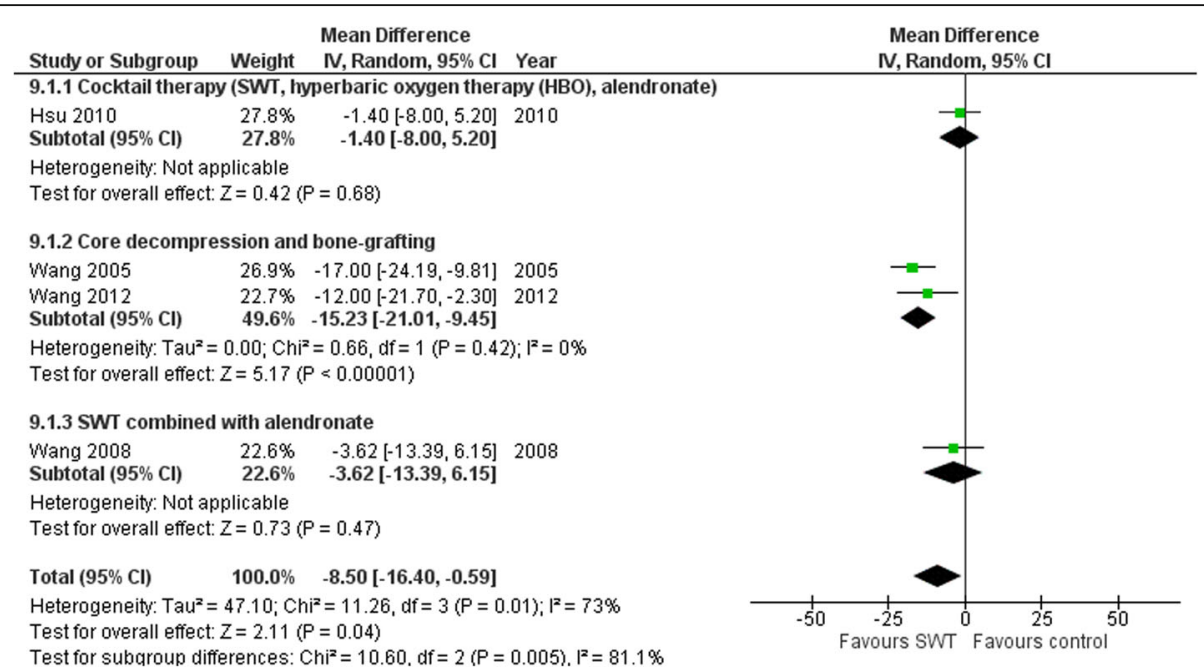

Fig. 10 Forest plot of effect of SWT vs control on femoral head necrosis lesion size (\%)

increased use of imaging outcomes in studies of this nature. This would assist in developing an improved understanding of the extent to which SWT has a therapeutic influence on pathophysiological processes in chronic musculoskeletal disorders.

\section{Conclusions}

The current review has identified some changes in imaging parameters of musculoskeletal conditions in response to SWT. Apparently, dosage parameters of SWT had no clear influence on the imaging outcomes. Also, the utilization of radiological guidance and local anesthesia is questionable. However, the size of lesion is found to be a potential predictor for change in response to SWT. Limitations related to imaging modality selection, timing of imaging and adequate reporting of imaging procedures were factors that influenced the conclusions that could be drawn from the review.

\section{Supplementary information}

Supplementary information accompanies this paper at https://doi.org/10. 1186/s12891-020-03270-w.

\section{Additional file 1}

Additional file 2

Additional file 3.

\section{Abbreviations}

ARCO: Association Research Circulation Osseous; BMC: Bone mineral content: BMD: Bone mineral density; BME: Bone marrow edema; $\mathrm{Cl}$ : Confidence interval; CT: Computed tomography; DEXA: Dual-energy x-ray absorptiometry; EFD: Energy flux density; SWT: Shockwave therapy; MD: Mean difference; MINORS: The methodological index for non-randomized studies; MRI: Magnetic resonance imaging; ONFH: Osteonecrosis of the femoral head; OR: Odds ratio; PF: Plantar fascia; PFT: Plantar fascia thickness:

RCT: Randomized controlled trial: SD: Standard deviation: US: Ultrasonography
Acknowledgements

We would like to acknowledge the assistance of Ms. Diana Blackwood,

Faculty Librarian, in helping us to develop an appropriate search strategy.

\section{Authors' contributions}

This research is a part of a thesis submitted as partial fulfilment of the requirements for the degree of Doctor of Philosophy (PhD). HA is a PhD candidate who was responsible for the conception and design, data synthesis and analysis, interpretation of data and drafting the manuscript. HA and SA screened the literature and assessed risk of bias independently. AW contributed to conception and design, supervised the project, validated the data, reviewed and edited the manuscript. JR, SM, EB and BP contributed to conception and design and reviewed and edited the manuscript. All authors read and approved the final manuscript.

Funding

None.

Availability of data and materials

All data generated or analyzed during this study are presented in this published article and its additional information files. Meta-analysis data can be requested from authors.

Ethics approval and consent to participate Not applicable.

Consent for publication

Not applicable.

\section{Competing interests}

The authors declare that they have no competing interests.

\section{Author details}

${ }^{1}$ School of Physiotherapy and Exercise Science, Curtin University, GPO Box U1987, Perth, WA, Australia. ${ }^{2}$ Physical Therapy department, Rehabilitation hospital, King Fahad Medical City, Riyadh, Saudi Arabia. ${ }^{3}$ College of Healthcare Science and Division of Tropical Health and Medicine, James Cook University, Townsville, QLD, Australia. ${ }^{4}$ College of Medicine and Dentistry, James Cook University, Townsville, Australia. ${ }^{5}$ HFRC, Nedlands, WA, Australia. 
Received: 6 January 2020 Accepted: 6 April 2020 Published online: 28 April 2020

\section{References}

1. Speed C. A systematic review of shockwave therapies in soft tissue conditions: focusing on the evidence. Br J Sports Med. 2014;48(21):1538-42.

2. Foldager CB, Kearney C, Spector M. Clinical application of extracorporeal shock wave therapy in orthopedics: focused versus unfocused shock waves. Ultrasound Med Biol. 2012;38(10):1673-80.

3. Korakakis V, Whiteley R, Tzavara A, Malliaropoulos N. The effectiveness of extracorporeal shockwave therapy in common lower limb conditions: a systematic review including quantification of patient-rated pain reduction. Br J Sports Med. 2018;52(6):387-407.

4. Verstraelen FU, In den Kleef NJ, Jansen L, Morrenhof JW. High-energy versus low-energy extracorporeal shock wave therapy for calcifying tendinitis of the shoulder: which is superior? A meta-analysis. Clin Orthop Relat Res. 2014;472(9):2816-25

5. Al-Abbad H, Simon JV. The effectiveness of extracorporeal shock wave therapy on chronic achilles tendinopathy: a systematic review. Foot Ankle Int. 2013:34(1):33-41.

6. Mani-Babu S, Morrissey D, Waugh C, Screen H, Barton C. The effectiveness of extracorporeal shock wave therapy in lower limb tendinopathy: a systematic review. Am J Sports Med. 2015;43(3):752-61.

7. Liao CD, Tsauo JY, Liou TH, Chen HC, Huang SW. Clinical efficacy of extracorporeal shockwave therapy for knee osteoarthritis: a systematic review and meta-regression of randomized controlled trials. Clin Rehabil. 2019;33(9):1419-30.

8. Hao Y, Guo H, Xu Z, Qi H, Wang Y, Lu C, Liu J, Yuan P. Meta-analysis of the potential role of extracorporeal shockwave therapy in osteonecrosis of the femoral head. J Orthop Surg Res. 2018;13(1):166.

9. Lou J, Wang S, Liu S, Xing G. Effectiveness of extracorporeal shock wave therapy without local anesthesia in patients with recalcitrant plantar fasciitis: a meta-analysis of randomized controlled trials. Am J Phys Med Rehabil. 2017;96(8):529-34.

10. van der Worp H, van den Akker-Scheek I, van Schie H, Zwerver J. ESWT for tendinopathy: technology and clinical implications. Knee Surg Sports Traumatol Arthrosc. 2013;21(6):1451-8.

11. Moya D, Ramon S, Schaden W, Wang CJ, Guiloff L, Cheng JH. The role of extracorporeal shockwave treatment in musculoskeletal disorders. J Bone Joint Surg Am. 2018;100(3):251-63.

12. Wang CJ. Extracorporeal shockwave therapy in musculoskeletal disorders. J Orthop Surg Res. 2012;7:11.

13. Holfeld J, Tepekoylu C, Kozaryn R, Urbschat A, Zacharowski K, Grimm M, Paulus P. Shockwave therapy differentially stimulates endothelial cells: implications on the control of inflammation via toll-like receptor 3. Inflammation. 2014;37(1):65-70.

14. Vertitas Health Innovation Ltd. Covidence systematic review software. Melborne: Veritas Heath Innovation Ltd.; 2018.

15. Higgins JP, Altman DG, Gotzsche PC, Juni P, Moher D, Oxman AD, Savovic J, Schulz KF, Weeks L, Sterne JA, et al. The Cochrane Collaboration's tool for assessing risk of bias in randomised trials. BMJ. 2011;343:d5928.

16. Slim K, Nini E, Forestier D, Kwiatkowski F, Panis Y, Chipponi J. Methodological index for non-randomized studies (minors): development and validation of a new instrument. ANZ J Surg. 2003;73(9):712-6.

17. Zeng $X$, Zhang Y, Kwong JS, Zhang C, Li S, Sun F, Niu Y, Du L. The methodological quality assessment tools for preclinical and clinical studies, systematic review and meta-analysis, and clinical practice guideline: a systematic review. J Evid Based Med. 2015;8(1):2-10.

18. The Nordic Cochrane Centre. Review Manager (RevMan). 5.3 ed; 2014

19. Gartner J, Simons B. Analysis of calcific deposits in calcifying tendinitis. Clin Orthop Relat Res. 1990:254:111-20.

20. Albert JD, Meadeb J, Guggenbuhl P, Marin F, Benkalfate T, Thomazeau H, Chales G. High-energy extracorporeal shock-wave therapy for calcifying tendinitis of the rotator cuff: a randomised trial. J Bone Joint Surg Br. 2007;89(3):335-41.

21. Cacchio A, Paoloni M, Barile A, Don R, De Paulis F, Calvisi V, Ranavolo A, Frascarelli M, Santilli V, Spacca G. Effectiveness of radial shock-wave therapy for calcific tendinitis of the shoulder: single-blind, randomized clinical study. Phys Ther. 2006;86(5):672-82.

22. Charrin JE, Noel ER. Shockwave therapy under ultrasonographic guidance in rotator cuff calcific tendinitis. Joint Bone Spine. 2001;68(3):241-4.
23. Cosentino R, De Stefano R, Selvi E, Frati E, Manca S, Frediani B, Marcolongo R. Extracorporeal shock wave therapy for chronic calcific tendinitis of the shoulder: single blind study. Ann Rheum Dis. 2003;62(3):248-50.

24. Daecke W, Kusnierczak D, Loew M. Long-term effects of extracorporeal shockwave therapy in chronic calcific tendinitis of the shoulder. J Shoulder Elb Surg. 2002;11(5):476-80.

25. De Boer FA, Mocking F, Nelissen EM, Van Kampen PM, Huijsmans PE. Ultrasound guided needling vs radial shockwave therapy in calcific tendinitis of the shoulder: a prospective randomized trial. J Orthop. 2017;14(4):466-9.

26. Del Castillo-Gonzalez F, Ramos-Alvarez JJ, Rodriguez-Fabian G, GonzalezPerez J, Jimenez-Herranz E, Varela E. Extracorporeal shockwaves versus ultrasound-guided percutaneous lavage for the treatment of rotator cuff calcific tendinopathy: a randomized controlled trial. Eur J Phys Rehabil Med. 2016;52(2):145-51

27. Farr S, Sevelda F, Mader P, Graf A, Petje G, Sabeti-Aschraf M. Extracorporeal shockwave therapy in calcifying tendinitis of the shoulder. Knee Surg Sports Traumatol Arthrosc. 2011;19(12):2085-9.

28. Gerdesmeyer L, Wagenpfeil S, Haake M, Maier M, Loew M, Wortler K, Lampe R, Seil R, Handle G, Gassel S, et al. Extracorporeal shock wave therapy for the treatment of chronic calcifying tendonitis of the rotator cuff: a randomized controlled trial. J Am Med Assoc. 2003;290(19):2573-80.

29. Hsu CJ, Wang DY, Tseng KF, Fong YC, Hsu HC, Jim YF. Extracorporeal shock wave therapy for calcifying tendinitis of the shoulder. J Shoulder Elb Surg. 2008;17(1):55-9.

30. Jakobeit C, Winiarski B, Jakobeit S, Welp L, Spelsberg G. Ultrasound-guided, high-energy extracorporeal shock-wave treatment of symptomatic calcareous tendinopathy of the shoulder. ANZ J Surg. 2002;72(7):496-500.

31. Kim YS, Lee HJ, Kim YV, Kong CG. Which method is more effective in treatment ofcalcific tendinitis in the shoulder? Prospective randomized comparison between ultrasound-guided needling and extracorporeal shock wave therapy. J Shoulder Elb Surg. 2014;23(11):1640-6.

32. Krasny C, Enenkel M, Aigner N, WIk M, Landsiedl F. Ultrasound-guided needling combined with shock-wave therapy for the treatment of calcifying tendonitis of the shoulder. J Bone Joint Surge Ser B. 2005;87(4):501-7.

33. Loew M, Daecke W, Kusnierczak D, Rahmanzadeh M, Ewerbeck V. Shockwave therapy is effective for chronic calcifying tendinitis of the shoulder. J Bone Joint Surg Ser B. 1999;81(5):863-7.

34. Loew M, Jurgowski W, Mau HC, Thomsen M. Treatment of calcifying tendinitis of rotator cuff by extracorporeal shock waves: a preliminary report. J Shoulder Elb Surg. 1995:4(2):101-6.

35. Moretti B, Garofalo R, Genco S, Patella V, Mouhsine E. Medium-energy shock wave therapy in the treatment of rotator cuff calcifying tendinitis. Knee Surg Sports Traumatol Arthrosc. 2005;13(5):405-10.

36. Pan PJ, Chou CL, Chiou HJ, Ma HL, Lee HC, Chan RC. Extracorporeal shock wave therapy for chronic calcific tendinitis of the shoulders: a functional and sonographic study. Arch Phys Med Rehabil. 2003;84(7):988-93.

37. Pigozzi F, Giombini A, Parisi A, Casciello G, Di Salvo V, Santori N, Mariani PP. The application of shock-waves therapy in the treatment of resistant chronic painful shoulder: a clinical experience. J Sports Med Phys Fitness. 2000;40(4):356-61.

38. Pleiner J, Crevenna R, Langenberger $H$, Keilani M, Nuhr M, Kainberger F, Wolzt M, Wiesinger G, Quittan M. Extracorporeal shockwave treatment is effective in calcific tendonitis of the shoulder. A randomized controlled trial. Wien Klin Wochenschr. 2004;116(15-16):536-41.

39. Rompe JD, Rumler F, Hopf C, Nafe B, Heine J. Extracorporal shock wave therapy for calcifying tendinitis of the shoulder. Clin Orthop Relat Res. 1995;321:196-201.

40. Rompe JD, Zoellner J, Nafe B. Shock wave therapy versus conventional surgery in the treatment of calcifying tendinitis of the shoulder. Clin Orthop Relat Res. 2001:387:72-82.

41. Sabeti-Aschraf M, Dorotka R, Goll A, Trieb K. Extracorporeal shock wave therapy in the treatment of calcific tendinitis of the rotator cuff. Am J Sports Med. 2005;33(9):1365-8.

42. Tornese D, Mattei E, Bandi M, Zerbi A, Quaglia A, Melegati G. Arm position during extracorporeal shock wave therapy for calcifying tendinitis of the shoulder: a randomized study. Clin Rehabil. 2011;25(8):731-9.

43. Daniel-Lucian D, Tatiana D. The effects of extracorporeal shockwave therapy (ESWT) for the plantar fasciitis in professional athletes. Ovidius Univ Ann Ser Phys Educ Sport/Sci Mov Health. 2013;13:595-602.

44. Bicer M, Hocaoglu E, Aksoy S, İnci E, Aktaș I, Aktaş I: Assessment of the efficacy of extracorporeal shockwave therapy for plantar fasciitis with magnetic resonance imaging findings. J Am Podiatr Med Assoc 2018, 108(2):100-105. 
45. Chew KTL, Leong D, Lin CY, Lim KK, Tan B. Comparison of autologous conditioned plasma injection, extracorporeal shockwave therapy, and conventional treatment for plantar fasciitis: a randomized trial. PM \& R. 2013; 5(12):1035-43.

46. Gerdesmeyer L, Schaden W, Besch L, Stukenberg M, Doerner L, Muehlhofer $H$, Toepfer A. Osteogenetic effect of extracorporeal shock waves in human. Int J Surg. 2015;24:115-9.

47. Hammer DS, Adam F, Kreutz A, Rupp S, Kohn D, Seil R. Ultrasonographic evaluation at 6-month follow-up of plantar fasciitis after extracorporeal shock wave therapy. Arch Orthop Trauma Surg. 2005;125(1):6-9.

48. Hocaoglu S, Vurdem UE, Cebicci MA, Sutbeyaz ST, Guldeste Z, Yunsuroglu SG. Comparative effectiveness of radial extracorporeal shockwave therapy and ultrasound-guided local corticosteroid injection treatment for plantar fasciitis. J Am Podiatr Med Assoc. 2017;107(3):192-9.

49. Lai TW, Ma HL, Lee MS, Chen PM, Ku MC. Ultrasonography and clinical outcome comparison of extracorporeal shock wave therapy and corticosteroid injections for chronic plantar fasciitis: a randomized controlled trial. J Musculoskelet Neuronal Interact. 2018;18(1):47-54.

50. Lee GP, Ogden JA, Cross GL. Effect of extracorporeal shock waves on calcaneal bone spurs. Foot Ankle Int. 2003;24(12):927-30.

51. Maki M, Ikoma K, Kido M, Hara Y, Sawada K, Ohashi S, Kubo T. Magnetic resonance imaging findings of chronic plantar fasciitis before and after extracorporeal shock wave therapy. Foot. 2017;33:25-8.

52. Moretti B, Garofalo R, Patella V, Sisti G, Corrado M, Mouhsine E, Sisti GL. Extracorporeal shock wave therapy in runners with a symptomatic heel spur. Knee Surg Sports Traumatol Arthrosc. 2006;14(10):1029-32.

53. Saber N, Diab H, Nassar W, Razaak HA. Ultrasound guided local steroid injection versus extracorporeal shockwave therapy in the treatment of plantar fasciitis. Alexandria J Med. 2012;48(1):35-42.

54. Sorrentino F, lovane A, Vetro A, Vaccari A, Mantia R, Midiri M. Role of highresolution ultrasound in guiding treatment of idiopathic plantar fasciitis with minimally invasive techniques. Radiol Med. 2008;113(4):486-95.

55. Ulusoy A, Cerrahoglu L, Orguc S. Magnetic resonance imaging and clinical outcomes of laser therapy, ultrasound therapy, and extracorporeal shock wave therapy for treatment of plantar fasciitis: a randomized controlled trial. J Foot Ankle Surg. 2017:56(4):762-7.

56. Vahdatpour B, Sajadieh S, Bateni V, Karami M, Sajjadieh H. Extracorporeal shock wave therapy in patients with plantar fasciitis. A randomized placebocontrolled trial with ultrasonographic and subjective outcome assessments. J Res Med Sci. 2012;17(9):834-8.

57. Yalcin E, Keskin Akca A, Selcuk B, Kurtaran A, Akyuz M. Effects of extracorporal shock wave therapy on symptomatic heel spurs: a correlation between clinical outcome and radiologic changes. Rheumatol Int. 2012;32(2):343-7.

58. Zhu F, Johnson JE, Hirose CB, Bae KT. Chronic plantar fasciitis: acute changes in the heel after extracorporeal high-energy shock wave therapy-observations at MR imaging. Radiology. 2005;234(1):206-10.

59. d'Agostino C, Romeo P, Lavanga V, Pisani S, Sansone V. Effectiveness of extracorporeal shock wave therapy in bone marrow edema syndrome of the hip. Rheumatol Int. 2014;34(11):1513-8.

60. Vulpiani MC, Vetrano M, Trischitta D, Scarcello L, Chizzi F, Argento G, Saraceni VM, MaVulli N, Ferretti A. Extracorporeal shock wave therapy in early osteonecrosis of the femoral head: prospective clinical study with long-term follow-up. Arch Orthop Trauma Surg. 2012;132(4):499-508.

61. Algarni AD, Al Moallem HM. Clinical and radiological outcomes of extracorporeal shock wave therapy in early-stage femoral head osteonecrosis. Adv Orthopedics. 2018;2018:7410246.

62. Chen JM, Hsu SL, Wong T, Chou WY, Wang CJ, Wang FS. Functional outcomes of bilateral hip necrosis: Total hip arthroplasty versus extracorporeal shockwave. Arch Orthop Trauma Surg. 2009;129(6):837-41.

63. Hsu SL, Wang CJ, Lee MSS, Chan YS, Huang CC, Yang KD. Cocktail therapy for femoral head necrosis of the hip. Arch Orthop Trauma Surg. 2010;130(1):23-9.

64. Ludwig J, Lauber S, Lauber HJ, Dreisilker U, Raedel R, Hotzinger H. Highenergy shock wave treatment of femoral head necrosis in adults. Clin Orthop Relat Res. 2001;387:119-26.

65. Wang CJ, Huang CC, Gung KC. Dosage effects of extracorporeal shockwave therapy for early hip necrosis. J Orthopaedic Res. 2016;35(Supplement 1): 179-86.

66. Wang CJ, Huang CC, Wang JW, Wong T, Yang YJ. Long-term results of extracorporeal shockwave therapy and core decompression in osteonecrosis of the femoral head with eight- to nine-year follow-up. Biom J. 2012;35(6):481-5.
67. Wang CJ, Ko JY, Chan YS, Lee MS, Chen JM, Wang FS, Yang KD, Huang CC. Extracorporeal shockwave for hip necrosis in systemic lupus erythematosus. Lupus. 2009;18(12):1082-6.

68. Wang CJ, Wang FS, Huang CC, Yang KD, Weng LH, Huang HY. Treatment for osteonecrosis of the femoral head: comparison of extracorporeal shock waves with core decompression and bone-grafting. J Bone Joint Surg Series A. 2005;87(11):2380-7.

69. Wang CJ, Wang FS, Yang KD, Huang CC, Lee MSS, Chan YS, Wang JW, Ko JY. Treatment of osteonecrosis of the hip: comparison of extracorporeal shockwave with shockwave and alendronate. Arch Orthop Trauma Surg. 2008:128(9):901-8

70. Wang C-J, Yang Y-J, Huang C-C. The effects of shockwave on systemic concentrations of nitric oxide level, angiogenesis and osteogenesis factors in hip necrosis. Rheumatol Int. 2011;31(7):871-7.

71. Rompe JD, Rosendahl T, Schollner C, Theis C. High-energy extracorporeal shock wave treatment of nonunions. Clin Orthop Relat Res. 2001;387:102-11.

72. Stojadinovic A, Kyle Potter B, Eberhardt J, Shawen SB, Andersen RC, Forsberg JA, Shwery C, Ester EA, Schaden W. Development of a prognostic naive bayesian classifier for successful treatment of nonunions. J Bone Joint Surg Am. 2011;93(2):187-94.

73. Vulpiani MC, Vetrano M, Conforti F, Minutolo L, Trischitta D, Furia JP, Ferretti A. Effects of extracorporeal shock wave therapy on fracture nonunions. Am J Orthop (Belle Mead NJ). 2012;41(9):E122-7.

74. Wang CJ, Chen HS, Chen CE, Yang KD. Treatment of nonunions of long bone fractures with shock waves. Clin Orthop Relat Res. 2001;387:95-101.

75. Cho NS, Park YH, Hwang JH, Yoon YC, Park MJ, Yoo JC, Park WH. Ultrasonographic features of the tennis elbow: which is more responsive for extracorporeal shock wave therapy? J Musculoskelet Pain. 2012;20(2):100-6.

76. Gunduz R, Malas FU, Borman P, Kocaoglu S, Ozcakar L. Physical therapy, corticosteroid injection, and extracorporeal shock wave treatment in lateral epicondylitis: clinical and ultrasonographical comparison. Clin Rheumatol. 2012;31(5):807-12

77. Gao F, Sun W, Li Z, Guo W, Kush N, Ozaki K. Intractable bone marrow edema syndrome of the hip. Orthopedics. 2015;38(4):e263-70.

78. Sansone $\mathrm{V}$, Romeo $\mathrm{P}$, Lavanga $\mathrm{V}$. Extracorporeal shock wave therapy is effective in the treatment of bone marrow edema of the medial compartment of the knee: a comparative study. Med Princ Pract. 2017;26(1):23-9.

79. Cheng Y, Zhang J, Cai Y. Utility of ultrasonography in assessing the effectiveness of extracorporeal shock wave therapy in Insertional Achilles Tendinopathy. Biomed Res Int. 2016;2016:2580969.

80. Buselli P, Coco V, Notarnicola A, Messina S, Saggini R, Tafuri S, Moretti L, Moretti B. Shock waves in the treatment of post-traumatic myositis Ossificans. Ultrasound Med Biol. 2010;36(3):397-409.

81. Kim JY, Lee JS, Park CW. Extracorporeal shock wave therapy is not useful after arthroscopic rotator cuff repair. Knee Surg Sports Traumatol Arthrosc. 2012;20(12):2567-72

82. D'Agostino C, Romeo P, Amelio E, Sansone V. Effectiveness of ESWT in the treatment of Kienbock's disease. Ultrasound Med Biol. 2011;37(9):1452-6.

\section{Publisher's Note}

Springer Nature remains neutral with regard to jurisdictional claims in published maps and institutional affiliations.

Ready to submit your research? Choose BMC and benefit from

- fast, convenient online submission

- thorough peer review by experienced researchers in your field

- rapid publication on acceptance

- support for research data, including large and complex data types

- gold Open Access which fosters wider collaboration and increased citations

- maximum visibility for your research: over $100 \mathrm{M}$ website views per year

At $\mathrm{BMC}$, research is always in progress.

Learn more biomedcentral.com/submissions 